\title{
Degradable vinyl polymers for biomedical applications
}

\author{
Vianney Delplace and Julien Nicolas*
}

\begin{abstract}
Vinyl polymers have been the focus of intensive research over the past few decades and are attractive materials owing to their ease of synthesis and their broad diversity of architectures, compositions and functionalities. Their carbon-carbon backbones are extremely resistant to degradation, however, and this property limits their uses. Degradable polymers are an important field of research in polymer science and have been used in a wide range of applications spanning from (nano)medicine to microelectronics and environmental protection. The development of synthetic strategies to enable complete or partial degradation of vinyl polymers is, therefore, of great importance because it will offer new opportunities for the application of these materials. This Review captures the most recent and promising approaches to the design of degradable vinyl polymers and discusses the potential of these materials for biomedical applications.
\end{abstract}

A fter more than 50 years of continuous growth, worldwide production of synthetic polymers in $2013^{1}$ was approaching $300 \mathrm{Mt}$, about half of which was obtained by free-radical polymerization (FRP) of vinyl monomers ( $45 \%$ of manufactured plastic materials and $\sim 40 \%$ of synthetic rubbers). FRP is certainly the most versatile and robust polymerization method because it requires undemanding synthetic conditions, is compatible with many organic solvents and water, and tolerates a wide variety of functional monomers; altogether this makes it relatively easy to translate to an industrial plant. The carbon-carbon backbones of vinyl materials (with the exception of poly(vinyl alcohol) ${ }^{2}$ ) resist degradation, which explains why the major industrial applications of vinyl polymers (for example, low-density polyethylene, poly(vinyl chloride), polystyrene, poly((meth)acrylic esters), poly(vinyl acetate) and fluoropolymers) are structural, such as packaging and building materials. But this very durability prevents hydrolysis or enzymatic degradation of the materials in biological environments, leading to waste disposal problems and environmental issues (of which the Great Pacific Garbage Patch is one of the most striking illustrations). Vinyl-based polymers are also employed in an increasing number of higher-added-value and specialty applications, especially in the area of microelectronics (for example, positive or negative resists in microlithography) and biomaterials (for example, drug delivery devices, tissue engineering scaffolds), for which degradability is often essential. Indeed, unlike polyesters and polypeptides, which contain labile groups in their main chain enabling cleavage into small pieces, the persistence of vinyl polymers employed for biomedical applications requiring degradable materials may cause toxicity and hamper their translation to clinical settings and, eventually, to the market.

This would be unfortunate in regards to the massive amount of work devoted to macromolecular engineering towards biomedical applications, especially since the advent of controlled radical polymerization $(\mathrm{CRP})^{3-6}$. This general term gathers several techniques that enable a high degree of control (for example, predictable molar mass, low molar mass distribution, high chain-end fidelity, and so on) and functionalization to be reached, while maintaining all the advantages of FRP (for example, ease of use, applicability to a broad range of vinyl monomers, tolerance to numerous processes such as bulk, solution, emulsion, dispersion, and so on). As a result of these advanced polymerization methods, the past few years have been marked by a surge in the design of innovative and more sophisticated vinyl materials. Some of these are intended for applications in different bio-related areas, such as drug delivery, diagnostics or tissue engineering. Despite significant advances from synthetic and conceptual points of view, some very encouraging results and even improved performance compared with traditional systems, the persistence and non-degradability of the vast majority of these materials may cause unwanted immune responses and toxicity, and eventually result in a deadlock regarding regulatory requirements and risk-benefit analysis.

Biodegradable polymers strictly refer to polymers that degrade by biological activity (that is, action of cells) via lowering of their molar masses, whereas the term degradable is preferred when the degradation results from the action of water (hydrolysis), whether it is in vitro or in vivo, enzymes in vitro (enzymatic degradation), or when the mechanism of chain scission is unknown or not proven as cell mediated ${ }^{7}$. The term bioresorbable is used when an injected polymer is fully assimilated or eliminated in vivo, for instance through renal or biliary pathways. Note that in vivo degradation or biodegradation are necessary prior to bioresorption for high molar mass polymers (a commonly accepted renal excretion limit is $\sim 40-60 \mathrm{kDa})^{8}$. Degradability is a key safety issue when choosing materials for specific biomedical applications (for example, drug delivery, tissue engineering, and so on). For instance, implantable biomedical devices must be biocompatible but stable in biological environments to perform their functions in the long term. Conversely, polymers for use as tissue-engineered implants or as nanocarrier materials for drug delivery applications need to degrade eventually and ideally be excreted so that no polymer remains in the body after treatment. The nature of the degradation products is also crucial since their toxicity will determine the ultimate biocompatibility of the materials. If in vivo degradation results in the release of naturally occurring monomeric components, they can also be metabolized by natural processes such as the Krebs cycle. This eliminates the risk of complications associated with the long-term presence of a foreign material. For tissue engineering applications, the degradation timescale should match with the tissue healing processes (from weeks to years), whereas 
a

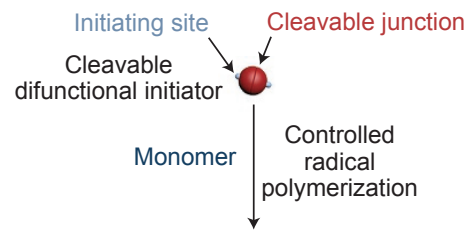

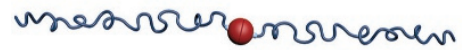

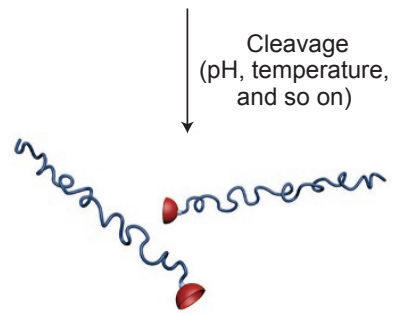

b

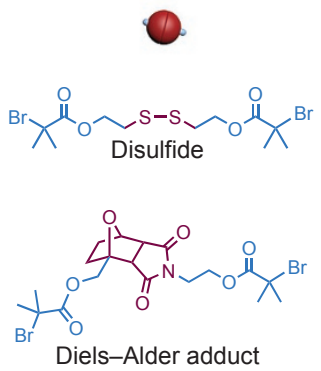

C

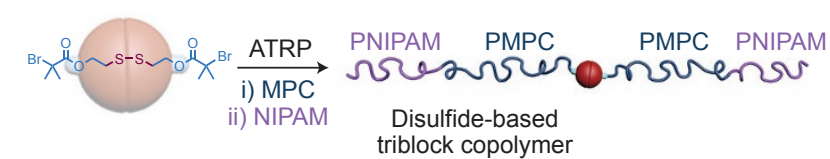

Self-assembly
in water

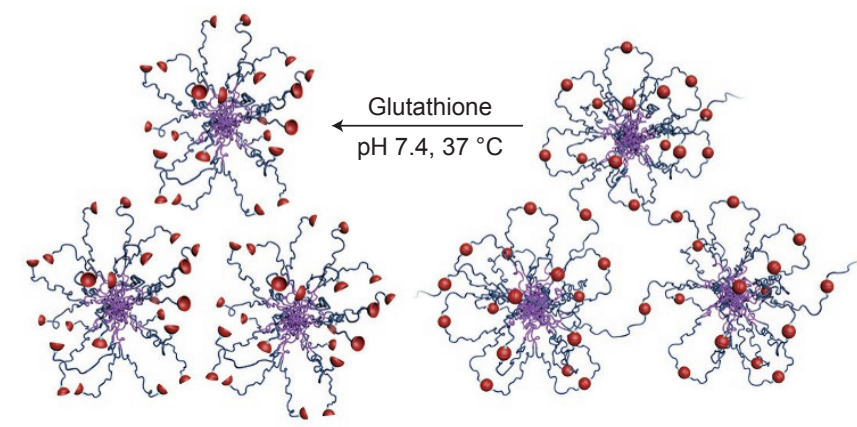

Free-flowing micelles at $37^{\circ} \mathrm{C}$

Free-standing micellar gel at $37^{\circ} \mathrm{C}$

Figure 1 | Cleavable difunctional initiators to prepare mid-chain degradable polymers. a, General synthetic pathway for the insertion of mid-chain degradable functionalities within a polymer backbone from a cleavable controlled radical polymerization (CRP) difunctional initiator. b, Representative cleavable difunctional atom-transfer radical polymerization (ATRP) initiators containing disulfide, Diels-Alder, o-nitrobenzyl ester and hemiacetal ester groups. c, Synthetic route to a disulfide-based thermo-responsive PNIPAM-b-PMPC-b-PNIPAM triblock copolymer gelator from a cleavable difunctional ATRP initiator. Panel c adapted from ref. 21, Wiley.

for drug delivery purposes, it should ideally start after the nanocarriers have reached the diseased site and proceed in parallel with the drug release (from hours and days to months). Once the nanocarriers have reached their target tissue, polymer degradation can facilitate drug delivery by enabling the release of physically encapsulated drugs.

In this very demanding context, strategies conferring degradability to vinyl materials are of paramount importance and may represent the missing link between the design of advanced vinyl materials and their safe use in biomedical applications. This Review discusses recent and promising synthetic strategies for degradable vinyl polymers and emphasizes potential candidates for biomedical applications. Even though each degradation mode may not find direct application in the biomedical field and pharmacokinetic aspects or potential toxicity issues are not discussed, all pathways leading to chain scission will be covered, with a particular focus on hydrolytic, enzymatic and reductive degradations, because they can mimic biological conditions (for example, $\mathrm{pH}$ found in certain cellular subcompartments or associated with some pathological situations, presence of specific enzymes or reducing agents in cells).

\section{Degradation of the polymer backbone}

The obvious approach to confer degradability to macromolecules is to introduce labile units within the polymer backbone. A small number of degradable links can cause a significant decrease in molar mass, providing they are homogeneously distributed along the chain. For instance, a single cleavable unit will enable a reduction of the number average molar mass $\left(M_{n}\right)$ by half after degradation. While multiple cleavable units are necessary for complete degradation, some applications require only partial degradation, and can be designed by combining degradable and non-degradable blocks.

Discrete insertion of main-chain degradable groups. The insertion of a single, typically central, degradable functionality into a vinyl polymer backbone became possible with the advent of CRP techniques, such as nitroxide-mediated polymerization (NMP), ${ }^{3}$ atom-transfer radical polymerization (ATRP $)^{4,5}$ and reversible addition-fragmentation chain-transfer polymerization (RAFT) ${ }^{6}$. The high degree of structural control and chain-end fidelity of CRP-derived polymers allows for the use of difunctional initiators containing a cleavable function ${ }^{9}$, followed by divergent chain growth, to position one degradable junction between two polymer blocks (Fig. 1a). In this instance, the disulfide bond appears to be the redox-sensitive linkage of choice (although the redox properties of selenium are receiving increasing interest ${ }^{10}$ ) for triggering cellular delivery applications by means of glutathione (GSH), one of the main reducing agents inside cells and the most abundant in the cytosol. However, it should be noted that the real biological situation is more complex, featuring subtle differences in redox potential within cells and a variety of different reducing agents ${ }^{11}$. To be translatable to the clinic, these characteristics should be considered in detail when developing redox-sensitive materials. Disulfidecontaining polymers can be designed by different CRP methods from various disulfide difunctional initiators ${ }^{9,12}$. Addition of GSH or dithiothreitol (DTT) is usually performed to demonstrate the cleavage. Other difunctional CRP initiators (whose cleavage conditions are perhaps less biologically relevant) were also considered (Fig. 1b), based on a Diels-Alder adduct (thermal degradation) $)^{13}$, a hemiacetal ester group (acid hydrolysis) ${ }^{14}$, or an $o$-nitrobenzyl moiety (photodegradation) ${ }^{15}$. Interestingly, the photocleavable nature of $o$-nitrobenzyl moieties enables the use of a non-invasive method of degradation that can be temporally and spatially controlled ${ }^{16,17}$. One can also take advantage of the susceptibility of trithiocarbonate to aminolysis as a means to centrally cleave polymers made from symmetrical trithiocarbonate RAFT agents ${ }^{18}$.

Owing to the simultaneity of the divergent chain growth, the use of cleavable difunctional initiators is restricted to the design of polymer blocks of the same nature, leading to symmetric architectures with a central degradable function. For instance, a single polymerization step will give a homopolymer, whereas consecutive polymerizations of different monomers will lead to an ABA triblock copolymer ${ }^{19,20}$. This has been illustrated by the preparation of disulfide-based poly $(N$-isopropyl acrylamide)-b-poly(2-methacryloyloxyethyl phosphorylcholine)b-poly( $N$-isopropyl acrylamide) (PNIPAM-b-PMPC-b-PNIPAM) 
triblock copolymer micelles forming a free-standing gel due to the establishment of disulfide bridges between the micelles. Nearly quantitative central cleavage by DTT caused irreversible dissolution of the micellar gel (Fig. 1c) ${ }^{21}$. This degradation was expected to be achieved under physiologically relevant conditions in the presence of naturally occurring oligopeptides, such as GSH. The method can also be adapted to multifunctional initiators containing degradable moieties to access complex architectures. For instance, tetrafunctional ATRP initiators with a central disulfide linkage and four ester groups bearing the initiating sites yield four-arm star polymers with two levels of degradability: reductive environments cleave the polymer into two symmetric parts, whereas alkaline conditions cut the four arms off 22 . This concept could be well adapted for drug delivery applications, as increasing the number of cleavable arms can result in unimolecular, degradable drug delivery vehicles, such as poly(ethylene glycol) (PEG)-based star-comb polymers ${ }^{23}$ or star-shaped polymers made of a $\beta$-cyclodextrin core and multiple polymethacrylate chains ${ }^{24}$.

Macromolecular coupling approaches are also convenient for preparing polymers with central cleavable groups embedded in them. A typical example are thermo-labile alkoxyamine functionalities, which cleave at high temperature and can be inserted by a variety of different techniques ${ }^{25-27}$. In a more biorelevant context, disulfide groups can also be positioned at the junction of $\mathrm{AB}$ diblock copolymers, thus enabling degradation under mild, reducing conditions. The methodology relies on the synthesis of two different ATRP-derived pyridyldisulfide (PDS) $\alpha$-functional polymers; one of them being further reduced to react with the one still containing a PDS functionality to form the disulfide bridge, whose cleavage has been shown in organic solution in the presence of $\mathrm{DTT}^{28}$. Owing to the broad variety of different PDS-terminated polymers that can be designed, multi-stimuli sensitive amphiphilic block copolymers have been prepared by connecting a temperature-responsive block and a $\mathrm{pH}$-responsive on $\mathrm{e}^{29}$. Supramolecular interactions can also efficiently connect two polymer chains together, thereby affording dynamic and reversible structures, whose cleavage can be governed by a broad range of stimuli (for example, redox potential, electric field, temperature, competitive ligand $)^{30}$. In the field of vinyl materials, most recent work stems from the design of well-defined polymers end-terminated by complementary moieties from the supramolecular toolbox (for example, hydrogen bonding, metal coordination, host-guest interactions) that are further reacted together ${ }^{31-34}$. The robustness of this approach has especially been illustrated by a onepot orthogonal self-assembly using two concomitant orthogonal hydrogen-bonding recognition pairs, leading to supramolecular $\mathrm{ABC}$ triblock copolymers ${ }^{35}$. Such a strategy may find applications as stimuli-responsive colloidal structures for drug delivery ${ }^{36}$, providing cleavage can be implemented in vivo. They can be formed and disassembled on demand on alternate ultraviolet-visible light exposure or electric stimulation, as shown for the latter with polystyrene$\beta$-cyclodextrin (PS- $\beta C D$ ) and poly(ethylene oxide)-ferrocene (PEO-Fc) supramolecular vesicles (Fig. 2) ${ }^{37}$. Despite encouraging results and proofs of concept, it would be important to test these systems in more relevant biological contexts to assess their in vitro and in vivo degradability.

Multiple insertions of main-chain degradable functionalities. These are required to achieve significant or complete degradation, which is particularly relevant for biomedical applications where the polymer material must eventually be excreted. For this purpose, macromolecular coupling approaches can be adapted to produce multisegmented degradable polymers by using difunctional precursors. For instance, although typical cleavage temperatures should be lowered for biomedical applications, alkoxyamine-containing multisegmented polymers can be obtained by atom-transfer nitroxide radical coupling (ATNRC) from ATRP-derived $\alpha, \omega$-dihalogenated polymers and dinitroxides ${ }^{38}$ or by nitrone-mediated radical coupling (NMRC) from similar polymer precursors ${ }^{39}$. Dinitroxides can also contain ester or disulfide moieties, thus conferring additional chemical and redox degradable properties, respectively, which could be well adapted for conferring degradability in vivo. On the other hand, disulfide-containing multisegmented polymers can readily be obtained by reacting together dithiol-terminated polymers under $\mathrm{FeCl}_{3}$ or $\mathrm{O}_{2}$ oxidation ${ }^{12,40}$. These materials are generally synthesized by ATRP (after nucleophilic substitution of the halide group of the corresponding dibromo-terminated polymers) ${ }^{12}$ or by RAFT (after aminolysis of the RAFT end groups $)^{40,41}$. Multiple disulfide functionalities can also be inserted by a polycondensation, step-growth procedure from a RAFT-derived macromonomer with a pyridyl disulfide end group, yielding polydisulfides with selective degradability in vitro in the presence of intracellular concentrations of $\mathrm{GSH}^{42}$.

An alternative to the formation of degradable junctions between polymer blocks is the incorporation of degradable moieties in the polymer backbone. This has been achieved by ATRP of divinyl monomers with dibromo compounds bearing disulfide or ketal functionalities ${ }^{43}$, or by multiple insertions of an enzymatically degradable peptide sequence (GFLG) in a vinyl polymer
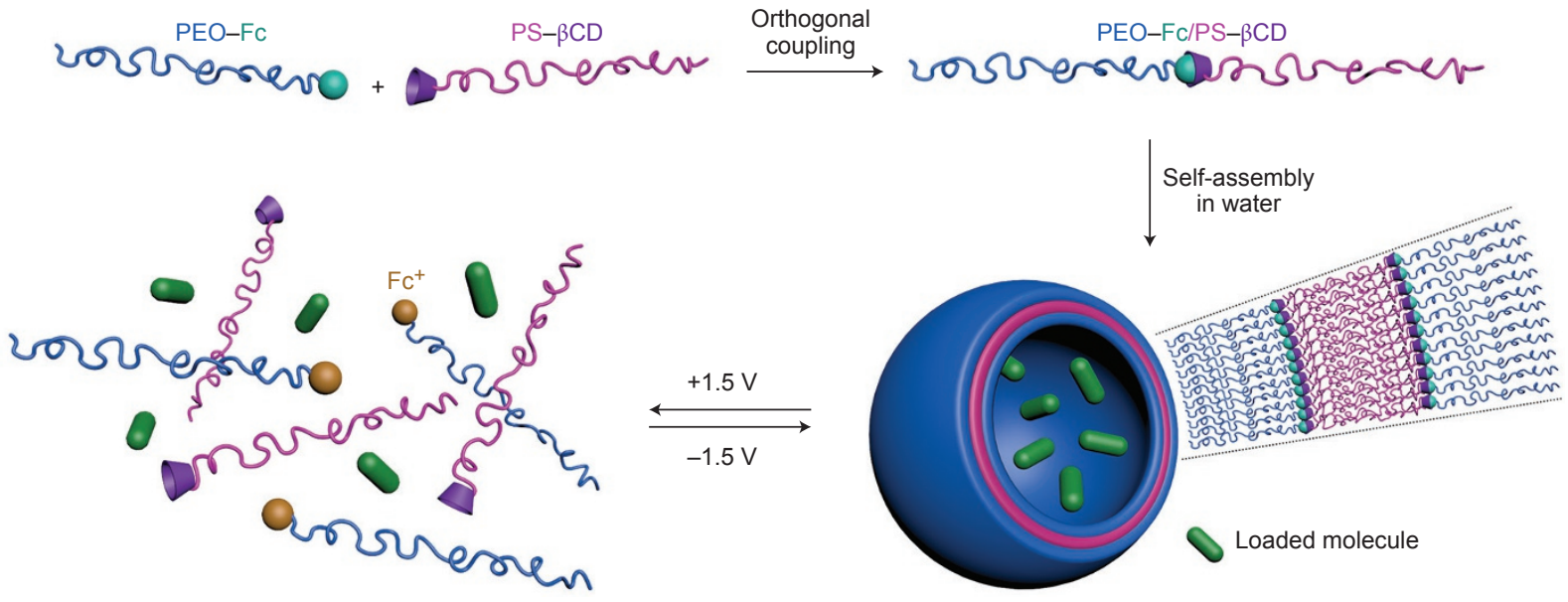

Figure 2 | Voltage-responsive polymer nanocapsules. End-functionalized polystyrene- $\beta$-cyclodextrin (PS- $\beta C D)$ and poly(ethylene oxide)-ferrocene (PEO-Fc) are able to orthogonally form PS- $\beta C D / P E O-F c$ diblock copolymers by $\beta C D$-Fc host-guest interaction. They can further self-assemble into supramolecular nanocapsules in aqueous solution whose assembly and disassembly can be reversibly controlled by means of an electric field through the association and disassociation of the central supramolecular junction. Figure adapted from ref. 37, ACS. 
chain. The latter approach was applied to water-soluble poly $(N$ (2-hydroxypropyl) methacrylamide) (PHPMA) coupled to doxorubicin (Dox $)^{44}$, which was the first polymer prodrug to enter clinical trials ${ }^{45}$. However, the non-degradable PHPMA-Dox prodrug showed marginal efficacy during phase II trials, possibly due to the relatively low molecular weight tested $(\sim 28 \mathrm{kDa})$. Conferring degradability to this system enabled the administration of higher molecular weight polymers, which were still excretable after degradation, and resulted in enhanced polymer prodrug accumulation in the tumour tissue by the enhanced permeability and retention effect. To do so, $a, \omega$-telechelic PHPMA chains prepared by RAFT polymerization were linked together by means of a difunctional GFLG sequence via copper(I)-catalysed azide-alkyne Huisgen cycloaddition (Fig. 3) or thiol-maleimide coupling ${ }^{46,47}$. This versatile method allows for additional GFLG sequences to be inserted in the polymer structure, either in the middle of each PHPMA chain by using a GFLG-containing difunctional RAFT agent, or on the side chain between each drug and the polymer scaffold to promote the drug release. This system was proven to be biodegradable and bioresorbable by means of pharmacokinetics and biodistribution studies $^{48}$ and has been applied to various anticancer agents (for example, Dox, gemcitabine, paclitaxel, platinum drugs ${ }^{48-52}$ and prostaglandin E1 as an anabolic agent in bone ${ }^{47}$. Star PHPMA-drug conjugates have also been prepared by linking PHPMA chains to poly(amidoamine) (PAMAM) dendrimers via GFLG sequences acting as degradable spacers ${ }^{53}$.

To circumvent the inherent constraints of consecutive macromolecular coupling, polyaddition and polycondensation reactions (for example, multistep processes, high dispersities), which may limit the applicability of the polymer, new polymerization concepts have emerged with the common feature of directly inserting multiple degradable functionalities in the course of the polymerization. This not only simplifies the synthetic pathway, but also gives more flexibility and robustness to the polymer design. In this respect, metal-catalysed step-growth radical polymerization of monomers possessing unconjugated $\mathrm{C}=\mathrm{C}$ and active $\mathrm{C}-\mathrm{Cl}$ bonds is an appealing strategy ${ }^{54}$. Such monomers can incorporate ester groups, leading to degradable vinyl homopolymers ${ }^{55}$, but they can also be copolymerized with traditional vinyl monomers using an ATRP catalyst (this method is then termed simultaneous chain- and step-growth radical polymerization) for novel degradable architectures ${ }^{54,56}$. Although this system suffers from broad molar mass distributions (which is inherent to step-growth polymerization), it has been applied to the design of self-degradable antibacterial polymers via the copolymerization of an amine-functionalized acrylate ${ }^{57}$. However, their in vivo degradability still needs to be demonstrated to make them potential candidates for biomedical applications requiring this feature. Interestingly enough, though molecular oxygen is frequently viewed as inimical to radical polymerization, its copolymerization with vinyl monomers by oxidative polymerization affords polyperoxides that degrade at high temperatures $\left(\sim 60-150{ }^{\circ} \mathrm{C}\right)^{58}$. These copolymerizations can also proceed under CRP conditions using reversible chain-transfer catalysed polymerization (RTCP) ${ }^{59}$ and produce biocompatible materials that can be cleaved by enzymatic degradation of the -O-O- sequence, which is encouraging regarding its potential degradability in $v i v o^{60}$. The use of polytrithiocarbonates, which can be seen as 'polyRAFT agents', can lead to well-defined polymers with multiple cleavable trithiocarbonate functionalities in the backbone after a single polymerization step ${ }^{61}$. The number of cleavable junctions is governed by the degree of functionality of the polyRAFT agent.

Despite these achievements, radical ring-opening polymerization (rROP) of cyclic monomers remains the radical polymerization technique of choice for incorporating degradable groups into the polymer backbone and enabling complete degradation. Thanks to its radical ring-opening mechanism, rROP possesses both the versatility of radical polymerization and the ability to introduce functional groups into the polymer backbone. The resulting polymers show low shrinkage, which is important for applications where constant volume is desirable, such as tooth fillings, coatings, and accurate moulding of electrical and electronic components. Several classes of cyclic monomers capable of undergoing rROP have been developed. Among them, cyclic ketene acetals (CKAs) are the moststudied family (Fig. 4a) and were the subject of extensive research by Bailey and co-workers in the early $80 \mathrm{~s}^{62}$. Although homopolymerization of CKAs is not straightforward, these monomers have aroused renewed interest over the past decade as comonomers to confer degradability to vinyl polymers via insertion of ester groups (Fig. 4b) ${ }^{63}$. The advantages offered by this approach are many: (i) different structures of CKAs can be synthesized (even mimicking traditional polyester repeat units once opened, such as polycaprolactone $(\mathrm{PCL})$ ), thus offering flexibility regarding the materials properties, although the number of efficient CKAs is still limited; (ii) despite unfavourable reactivity ratios requiring a large excess of CKAs in the
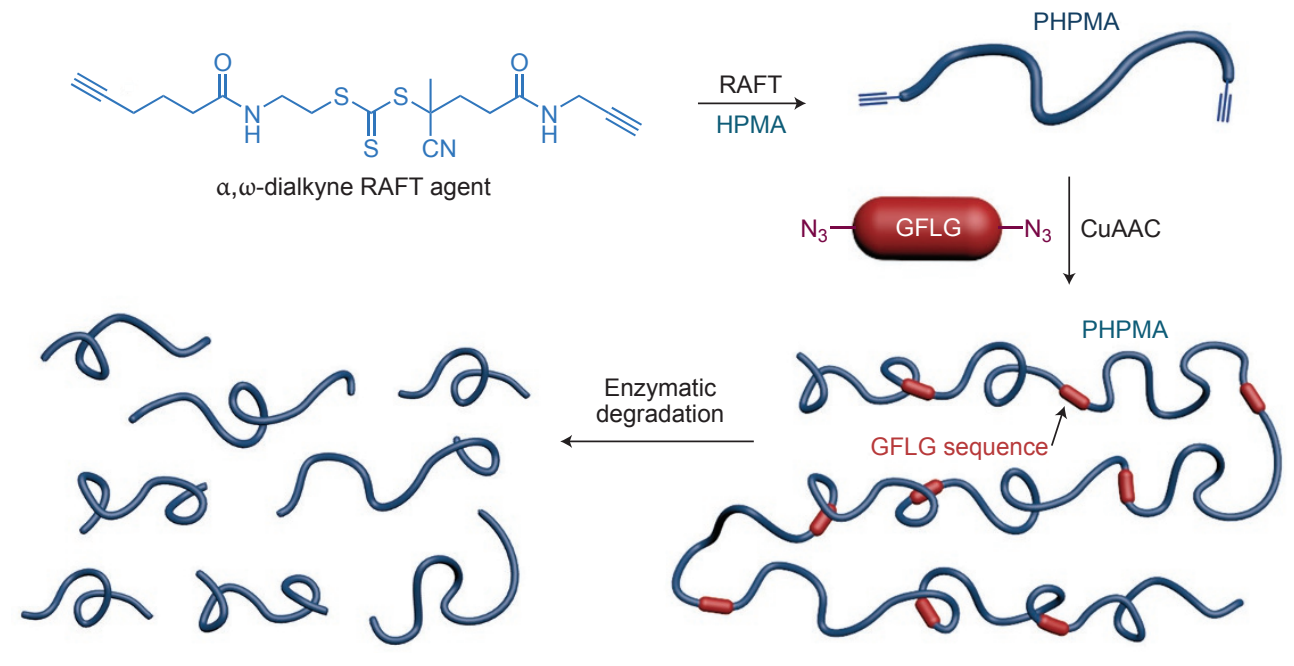

Figure 3 | Multisegmented polymer comprising biodegradable sequences in the main chain. Synthesis of multisegmented poly( $N$-(2-hydroxypropyl) methacrylamide) (PHPMA) copolymer by a combination of reversible addition-fragmentation chain-transfer (RAFT) polymerization of HPMA followed by copper-catalysed azide-alkyne Huisgen cycloaddition (CuAAC) between the resulting $\alpha, \omega$-dialkyne PHPMA and $a, \omega$-diazide GFLG peptide sequence. Figure adapted from ref. 46, ACS. 
a
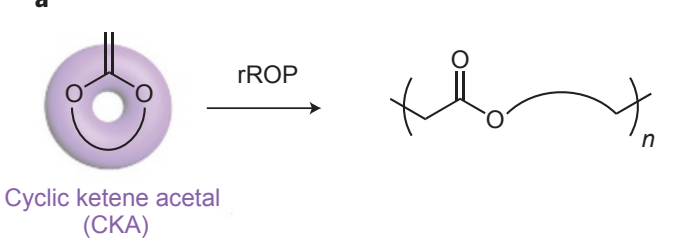

(CKA)

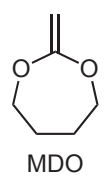

MDO
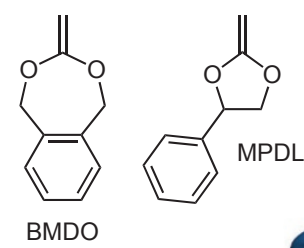

BMDO నs

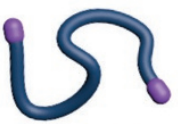

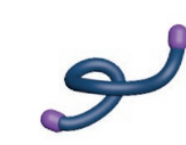

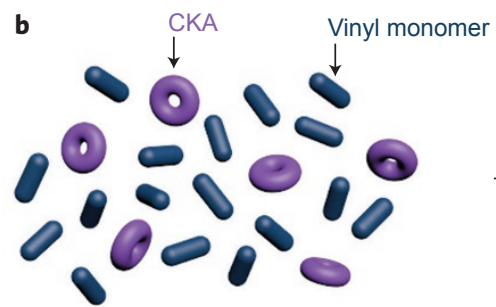

Copolymerization

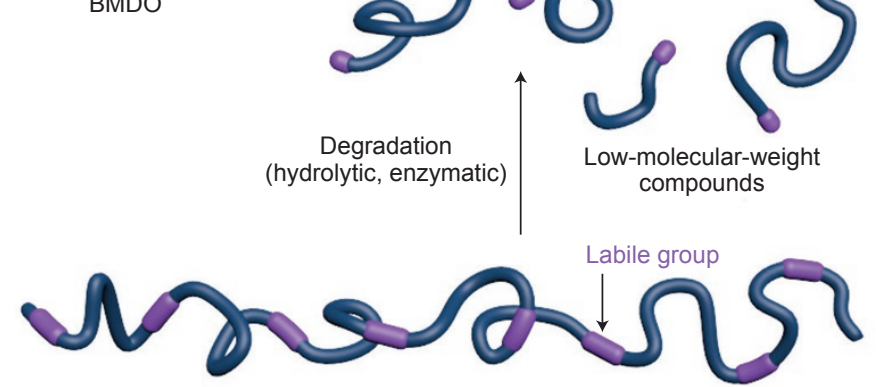

Copolymer containing labile groups in the main chain

Figure 4 | Radical ring-opening polymerization (rROP) to insert ester groups in the polymer backbone. a, rROP of cyclic ketene acetals (CKAs) and structures of three representative CKAs (MDO = 2-methylene-1,3-dioxepane; BMDO = 5,6-benzo-2-methylene-1,3-dioxepane; $\mathrm{MPDL}=$ 2-methylenephenyl-1,3-dioxolane). $\mathbf{b}$, Synthesis of a copolymer comprising multiple ester functions by radical copolymerization between a vinyl monomer and a CKA polymerized by rROP.

comonomer feed, these monomers can be copolymerized with several traditional vinyl monomers both by FRP and CRP techniques, for designing well-defined, complex macromolecular architectures, such as block, grafted or star copolymers; and (iii) CKAs are randomly distributed into the copolymer backbone, which enables finetuning of the degradability of the materials simply by adjusting the monomer feed. The flexibility of this approach has been illustrated by the synthesis of a variety of different copolymer structures ${ }^{64-69}$, which are intended to find applications in drug delivery, tissue engineering or hydrophobic coatings. Other families of cyclic monomers for performing rROP have been investigated as well, but to a lesser extent. Besides several methylene-dioxolane derivatives that lead to the insertion of ketones with potential photodegradability ${ }^{70}$, cyclic allylic sulfides have shown promising results, especially since they can be used to incorporate ester, thioester and disulfide functionalities into the polymer backbone under controlled radical copolymerization conditions with traditional vinyl monomers ${ }^{71,72}$. Although rROP-derived polymers are currently attracting renewed interest, their degradability in vivo has not yet been shown. Current examples only refer to hydrolytic and in vitro enzymatic degradations, as well as degradation by composting ${ }^{67}$.

While radical polymerization has captured most of the attention, living cationic polymerization can also be used to copolymerize naturally occurring aldehydes and vinyl ethers for the synthesis of alternating, degradable copolymers bearing acetal functions ${ }^{73}$. It is noteworthy that, due to the alternating structure, degradation on acid hydrolysis is observed down to monomer-unit molecular weight. Also, the broad structural diversity of vinyl ethers gave access to a wide range of different copolymers exhibiting water solubility and $\mathrm{pH}$ - and thermo-responsiveness ${ }^{74}$.

Copolymers with non-vinyl degradable blocks. An efficient way of producing degradable vinyl-based materials consists of combining a CRP technique with a polymerization method leading to well-known degradable polymer segments, such as ring-opening polymerization (ROP), either of lactones or $N$-carboxyanhydrides. Although here the vinyl block is persistent, the resulting copolymer will contain polyester or polypeptide chains that can be degraded in vivo hydrolytically or by specific enzymes, thus ensuring substantial degradability of the whole material. An important advantage is that synthetic polyesters, such as poly(lactic acid), poly(lactic-co-glycolic acid) and PCL, are well-known US Food and Drug Administration-approved biodegradable materials. By varying the combination of polymerization methods, the nature of the monomers and the synthetic strategies, a large range of degradable architectures with unique properties can be designed owing to structurally different blocks. For example, hydroxyl or amine functional CRP initiators can be used for combining ROP and CRP by divergent chain growth to produce partially degradable diblock copolymers ${ }^{75-78}$, and for some of them in a one-step process providing appropriate conditions are applied (Fig. 5a) ${ }^{79}$. This strategy can be extended to ABA triblock copolymers comprising a degradable central block by starting from a difunctional polyester macroinitator for CRP ${ }^{80,81}$. From such block copolymers, potential biomedical applications have been suggested, including nanoparticles ${ }^{82}$, micelles ${ }^{75,83}$, nanocages ${ }^{84}$ and porous membranes ${ }^{81}$ for drug delivery, and hydrogels ${ }^{80}$ for tissue engineering. Other partially degradable morphologies have also been investigated. For instance, a range of selectively degradable core crosslinked star (CCS) copolymers that can find applications in drug delivery or membrane formation have been synthesized, including arm-degradable CCS, partially arm-degradable CCS and core-degradable CCS polymers

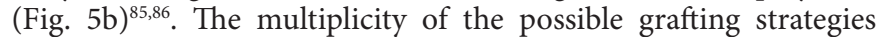
enables great variation of the final macromolecular structure and ensures different levels of degradation. A typical example are bottle-brush macromolecules, which received considerable attention due to their potential for intramolecular nanoengineering, such as templates for inorganic nanoparticles or nanowires. Three efficient pathways have been developed for the synthesis of bottle-brushes comprising degradable segments: (i) the 'grafting from' method, for which vinyl chains are grown under ATRP conditions from polymer scaffolds based on halogenated ROP monomers, such as $\alpha$-chloro- $\varepsilon$-caprolactone $(\alpha-\mathrm{Cl}-\varepsilon$-CL) or $N$ - $\varepsilon$-bromoisobutyrylL-lysine $\mathrm{N}$-carboxyanhydride (Br-Lys-NCA) ${ }^{87}$; (ii) the opposite 'grafting from' method, which uses hydroxyl-decorated vinyl backbones suitable for initiating ROP 88 ; and (iii) the 'grafting through' method, which relies on the CRP of degradable macromonomers, such as (meth)acrylate-terminated poly(D,L-lactide) and PCL ${ }^{89}$. By combining and permuting different coupling and polymerization (ROP and CRP) steps, all kinds of partially degradable vinyl-based brushed architectures are virtually accessible ${ }^{88,90}$. Even though all these examples reported vinyl architectures comprising segments 

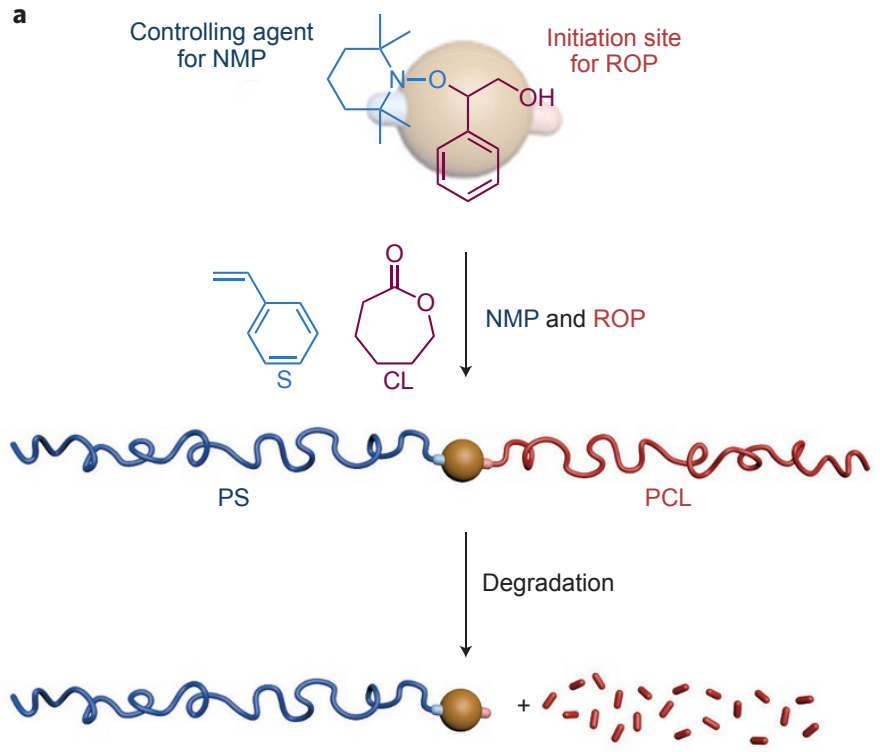

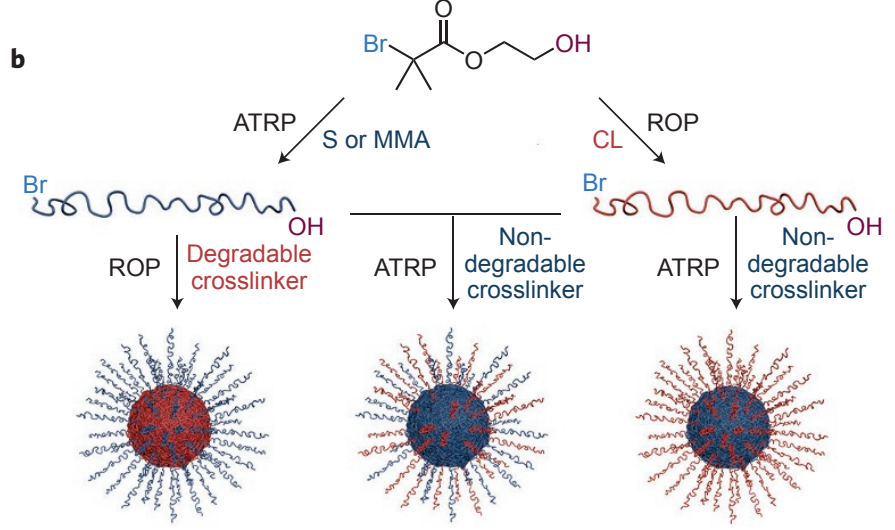

Core-degradable CCS Partially arm-degradable CCS Arm-degradable CCS

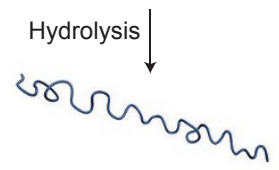

Hydrolysis

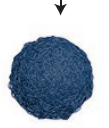

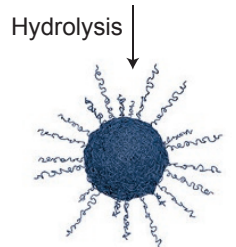

Figure 5 | Copolymers with non-vinyl degradable blocks from heterodifunctional initiators. a, Simultaneous nitroxide-mediated polymerization (NMP) of styrene (S) and ring-opening polymerization (ROP) of $\varepsilon$-caprolactone (CL) from a hydroxyl functional alkoxyamine for a one-step approach to $\mathrm{PS}$-b-PCL diblock copolymers. b. Synthesis of a selectively degradable core crosslinked star (CCS) polymer from a hydroxyl functional atom-transfer radical polymerization (ATRP) initiator and subsequent hydrolysis to remove the labile component (MMA = methyl methacrylate). Panel $\mathbf{b}$ adapted from ref. 85 , ACS.

based on well-known degradable polyesters and polypeptides, only hydrolytic degradation has been performed and their in vivo degradation or biodegradation still needs to be confirmed. The polycondensation of disulfide-containing diacids and diols combined with ATRP also received some attention as a means to prepare reductively degradable block copolymer nanocarriers ${ }^{91}$.

\section{Degradation of the polymer side chain}

Side-chain cleavage of vinyl polymers is an important lever to confer degradation, especially to nanoparticulate systems. Even though the average polymer chain length is not affected, the substantial structural modifications that occur can either make the polymer hydrophilic, thus leading to nanoparticle degradation, or trigger the disassembly of colloidal structures. This facilitates their excretion from the body and thus makes them well adapted for drug delivery applications.
Degradation leading to water solubility. Certain acrylic polymers can undergo ester side-chain hydrolysis in vivo by means of esterases to become water soluble, which makes their excretion possible. The only two polymer classes that fall into this category are poly(methylidene malonate) and poly(alkyl cyanoacrylate) (PACA) (Fig. 6a). The presence of two electron-withdrawing groups in the a-carbon to the double bond makes the corresponding monomers extremely reactive towards nucleophiles, such as anions or weak bases, resulting in quasi-instantaneous anionic polymerization $^{92}$. Initially used as surgical glue (Dermabond) or super glue for do-it-yourself activities (Loctite), PACA polymers from long alkyl cyanoacrylate monomers (for example, $n$-butyl, isobutyl, isohexyl, and so on) have been extensively investigated as biodegradable drug nanocarrier materials since the 80 s (Fig. 6b) ${ }^{93}$. A typical example is the development of Dox-loaded poly(isohexyl cyanoacrylate) a

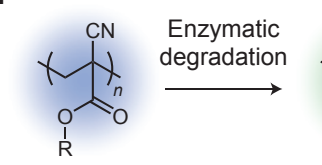

Hydrophobic polymer ( $R$ = alkyl chain)

b<smiles>C=C(C#N)C(=O)OCCCC</smiles><smiles>C=C(C#N)C(=O)OCC(C)C</smiles>

IBCA

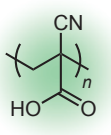

Hydrophilic polymer

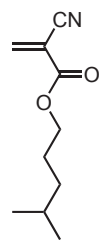

IHCA

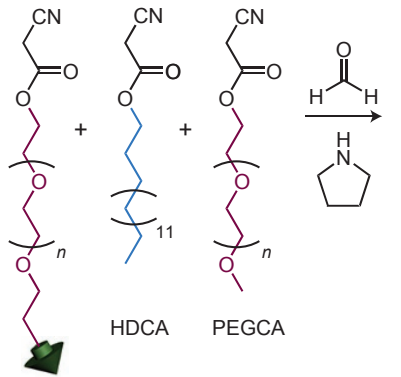

Ligand (imaging, targeting)

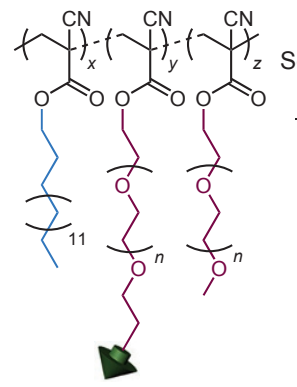

Functionalized amphiphilic copolymer

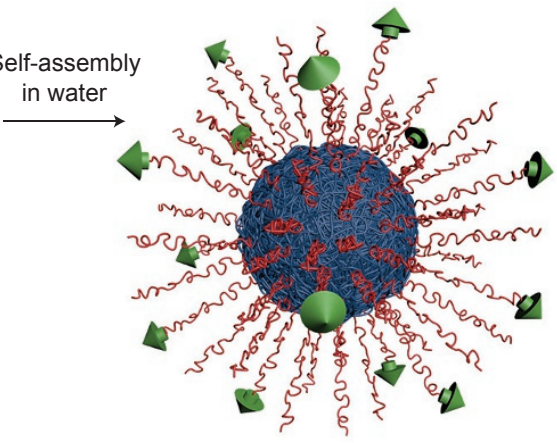

Functionalized nanoparticles

Figure 6 | Poly(alkyl cyanoacrylate) as a biodegradable polymer. a, General structure and side-chain degradation pathway of poly(alkyl cyanoacrylate) polymers. b, Structure of three representative alkyl cyanoacrylate monomers ( $n B C A=n$-butyl cyanoacrylate; IBCA = isobutyl cyanoacrylate; IHCA = isohexyl cyanoacrylate). c, Design of functionalized, PEGylated nanoparticles by tandem Knoevenagel condensation-Michael-type addition from hexadecyl cyanoacetate (HDCA), poly (ethylene glycol) cyanoacetate (PEGCA) and its functionalized counterpart. 
nanoparticles obtained by anionic emulsion polymerization that are currently under phase III clinical trials for the treatment of primary liver cancer ${ }^{94}$. A downside to the high reactivity of these monomers is the notable difficulty to design engineered and functionalized (co)polymers. This obstacle can be bypassed, however, by using cyanoacetate derivatives as monomer precursors. By tandem Knoevenagel condensation-Michael addition, the corresponding cyanoacrylate monomers are obtained in situ and can be further polymerized (Fig. 6c). From a mixture of hexadecyl cyanoacetate, rhodamine cyanoacetate and PEG cyanoacetate, this method enabled the formation of amphiphilic fluorescent copolymers that selfassembled into narrowly dispersed PEGylated nanoparticles, used for cell imaging and tracing purposes ${ }^{95,96}$. Increased sophistication was witnessed by positioning at the extremity of the PEG chains some targeting ligands directed against overexpressed cancer cell receptors or the amyloid- $\beta$ peptide. This resulted in a versatile, nanoparticulate platform that exhibited stealth, fluorescent and targeting abilities against cancer and Alzheimer's disease ${ }^{97}$.

Cleavage triggering colloidal disassembly. Side-chain cleavage can also activate rapid colloidal disassembly. For instance, amphiphilic diblock copolymers comprising a hydrophobic poly(methacrylate) block bearing pendent disulfide linkages formed drug-loaded micelles and released their load in aqueous solution due to complete disintegration in response to excess of thiols ${ }^{98}$. The system is versatile, as an equimolar thiol amount led to core crosslinked nanoparticles via thiol-disulfide exchange reactions. Cleavable side chains may also be attractive for gene delivery applications. Brushed copolymers composed of a methacrylate backbone onto which poly(2-(dimethylamino)ethyl methacrylate) (PDMAEMA) chains were grafted via a hydrolyzable carbonate linker were shown ${ }^{99}$ to condense plasmid DNA into positively charged polyplexes of about 100-200 nm. Hydrolysis in physiological conditions $(\mathrm{pH} 7.4$ at $37^{\circ} \mathrm{C}$ ) triggered the disintegration of the polyplexes and the subsequent release of plasmid DNA. This material is presumed to be bioresorbable as the molecular weight of the degradation product was found to be very close to that of the starting PDMAEMA, which is probably below the renal excretion limit. The method has been adapted to the grafting of cleavable PDMAEMA brushes onto polyaspartamide backbones ${ }^{100}$.

\section{Connecting polymer chains via degradable junctions}

Instead of introducing degradable units in the polymer backbone or side chains, an appealing alternative consists of connecting polymer chains together via degradable or reversible linkages. This results in a variety of different architectures and structures that can be degraded at the chain connection sites. They can therefore find applications in the biomedical field, particularly drug delivery applications. If bioresorbable materials are also targeted, the remaining chains should be small enough to be below the renal excretion limit and be cleared in vivo, unless they also contain main-chain degradable moieties.

Degradable crosslinkers. A straightforward strategy to connect polymer chains together relies on the use of difunctional vinyl comonomers, usually termed crosslinkers. In the field of degradable vinyl polymers, a significant amount of work has been devoted to the development of crosslinkers with labile functionalities embedded to implement the degradability of the resulting materials. It offers significant advantages over other approaches: (i) a broad range of different crosslinkers are readily accessible by varying the nature of both the polymerizable group and the degradable functionality; (ii) the resulting polymer is rather easy to obtain as the synthesis only requires the addition of the crosslinker in the reaction medium; and (iii) depending on the experimental conditions, different architectures and nano-objects can be prepared.

For instance, star polymers with a degradable core are obtained via the 'arm first' method, which either generates the core of the star macromolecule by coupling monofunctional 'living' polymeric chains with cleavable crosslinkers, ${ }^{101}$ or alternatively by direct copolymerization of a macromonomer with a degradable crosslinker. The latter approach was illustrated by the copolymerization of a PEG-based macromonomer with a disulfide methacrylate

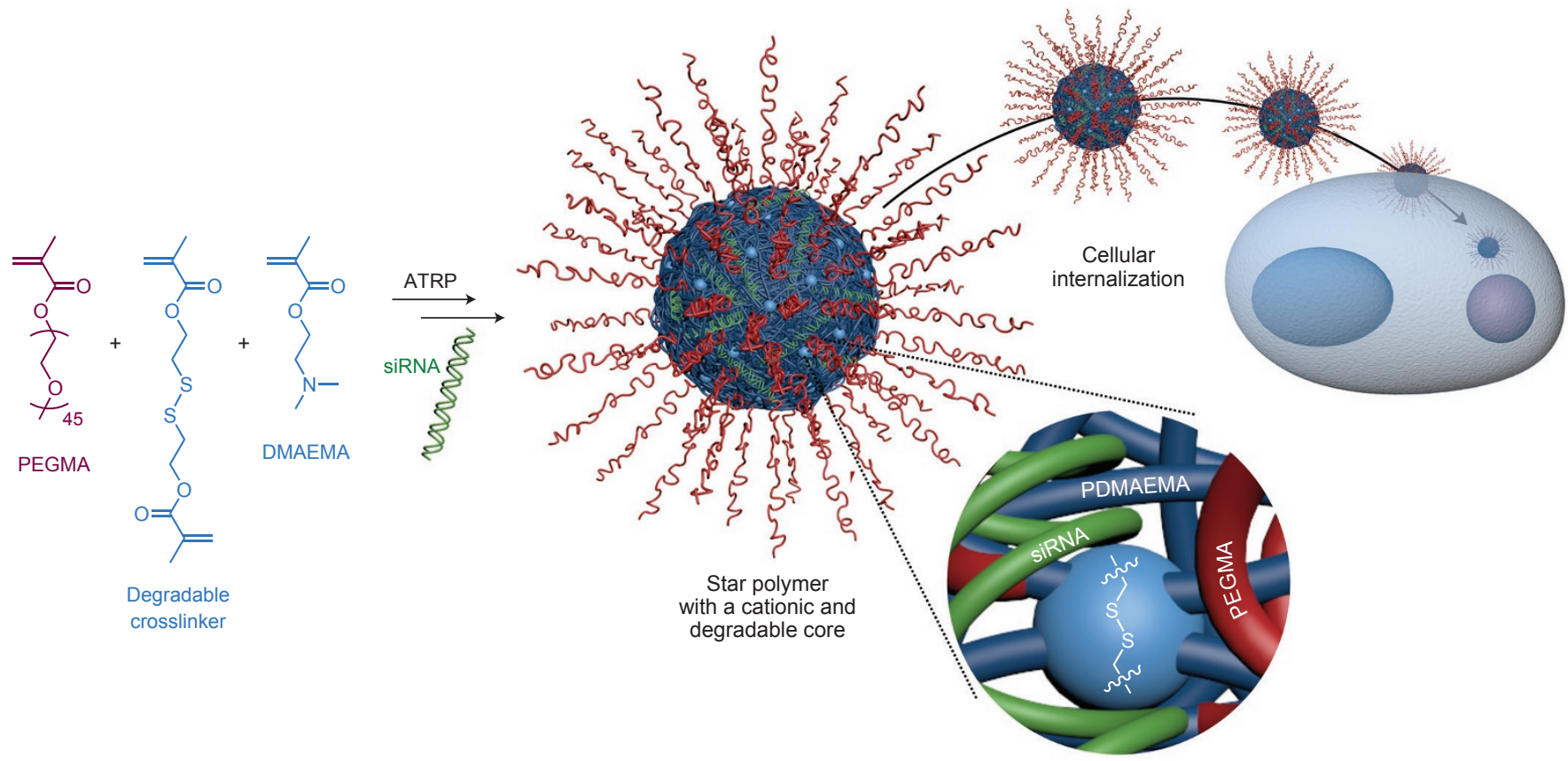

Figure 7 | Using a cleavable crosslinker to prepare degradable star polymers. Copolymerization of poly(ethylene glycol)methyl ether methacrylate (PEGMA), 2-(dimethylamino)ethyl methacrylate (DMAEMA) and bis(2-methacryloyloxyethyl) disulfide as a cleavable crosslinker to prepare biocompatible PEG-based star polymers with a cationic and degradable PDMAEMA core via atom-transfer radical polymerization (ATRP) and the 'arm first' method for the delivery of siRNA. 


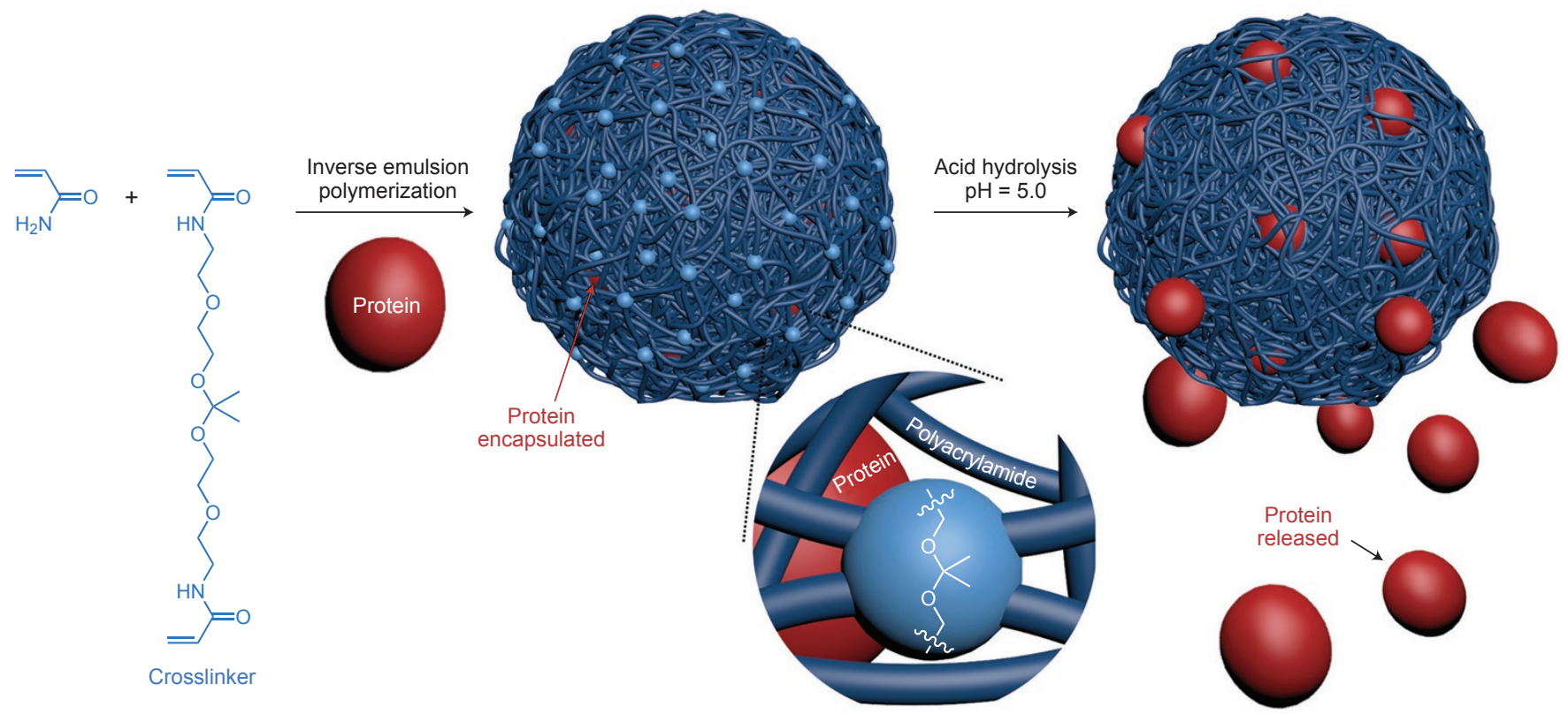

Figure 8 | Acid-degradable microparticles from a cleavable crosslinker. Inverse emulsion copolymerization of acrylamide and an acetal crosslinker to prepare acid-degradable crosslinked polyacrylamide microparticles for protein-based vaccines. The protein is released through degradation of the crosslinker.

and 2-(dimethylamino)ethyl methacrylate (DMAEMA) as a cationic vinyl monomer. Star copolymers for the delivery of small interfering RNA (siRNA) were prepared and degraded into individual polymeric chains in aqueous solution under reducing conditions (Fig. 7) ${ }^{102}$.

Similar difunctional vinyl comonomers can also lead to (hyper) branched structures, which have recently received much attention due to numerous applications in which a large density of functionalities is desirable, including reactive adhesives and coatings, imaging, and drug delivery. Conferring them with degradable features appears essential for drug-delivery purposes. Interestingly, the branching process is better controlled if the synthesis is performed under CRP conditions. For example, when a redox sensitive crosslinker is employed in combination with ATRP or RAFT, the degraded polymer obtained after reduction by DTT in organic solution exhibits a nearly similar molecular weight distribution as the linear counterpart obtained in the absence of any divinyl reagent $^{103,104}$. It was also shown that careful selection of the primary chain length enabled the production of poly(hydroxyethyl methacrylate) (PHEMA) fibres by electrospinning that could be further cleaved into linear PHEMA ${ }^{105}$. This may have biomedical applications considering that PHEMA can be degraded by macrophages ${ }^{106}$.

If experimental conditions enable the gel point to be exceeded, fully crosslinked 3D polymeric networks with elastic properties are obtained. Among these, hydrogels have attracted most attention for controlled drug delivery applications and as scaffold materials, on account of their similarity to soft biological tissues and their highly tunable mechanical properties. Recent achievements in degradable hydrogels employed FRP ${ }^{107-109}$ and CRP $80,107,110-113$ techniques. CRP generally provides more homogeneous structures compared with those obtained by FRP and also lead to predetermined molecular weights and narrow molecular weight distributions of the decomposed fragments ${ }^{107}$. This is particularly relevant because it reduces the chance of non-excretable residues being released upon degradation. This was especially illustrated by the ATRP of HEMA ${ }^{80}$, NIPAM $^{112}$ and DMAEMA ${ }^{107}$ in the presence of a divinyl macromolecular crosslinker based on PCL, leading to hydrogels with different mechanical and physicochemical properties. Hydrolytic degradation was demonstrated and its rate was controlled by the crosslinking density, the PCL chain length and the primary vinyl chain length ${ }^{80}$. Original strategies leading to more sophisticated hydrogels have also been reported, among which are the use of a divinyl tetrapeptide sequence (CYKC) as a crosslinker for the preparation of protease-responsive hydrogels ${ }^{114}$, the incorporation of cleavable poly(oligo(ethylene glycol) methyl ether methacrylate) (POEGMA) crosslinked nanoparticles into hydrogels ${ }^{113}$ and the crosslinking of PVA macromonomers ${ }^{115}$. Despite all these promising results, more investigations are needed to demonstrate the in vivo degradability of such systems to make a critical step forward for applications in drug delivery or tissue engineering. The slow hydrolytic degradation of the ester bonds of PEG diacrylate-based hydrogels may also open new opportunities in this field ${ }^{116}$.

When the copolymerization between a traditional vinyl monomer and a degradable divinyl crosslinker is performed in aqueous dispersed media (for example, dispersion, suspension, emulsion, and so on), degradable particulate systems that can find application as drug carriers are obtained. A typical example are aciddegradable crosslinked polyacrylamide microparticles for vaccine development, synthesized by inverse free-radical (micro)emulsion polymerization in the presence of acetal crosslinkers (Fig. 8) 109,117-120. $^{\text {. }}$ These particles allow for plasmid and protein encapsulation and can degrade under the mildly acidic conditions found in the phagosomes of antigen-presenting cells. By using ovalbumin as a model antigen, the system performances were further enhanced by the coencapsulation $^{121}$ or surface attachment ${ }^{122}$ of immunostimulatory CpG DNA to better mimic actual pathogens, and by the design of cell-penetrating peptide-modified microparticles to improve cell uptake $^{123}$. Nanosized hydrogels (often termed nanogels), synthesized by inverse miniemulsion ATRP of OEGMA and a reducible divinyl crosslinker, also represent a promising reductively degradable system ${ }^{124}$. They have demonstrated not only high drug loading capabilities and efficient release upon degradation ${ }^{125,126}$, but also the capacity to be surface functionalized with biologically active moieties ${ }^{126}$. Acetal crosslinkers can also be used on preformed block copolymer nanoparticles obtained by self assembly in aqueous solution ${ }^{127}$, which represents a valuable alternative to direct polymerization in aqueous dispersed systems. The simplicity of these strategies and the efficiency of hydrolytic or reductive degradations are particularly appealing, but the in vivo degradability of the resulting carriers is still to be demonstrated. 
Other strategies to insert degradable junctions. Many additional pathways have been proposed to connect polymer chains together in a degradable fashion. The use of degradable inimers (a class of molecules having both an initiator and monomer fragment) is appealing for its simplicity and robustness, as it readily enables the production of degradable hyperbranched structures. This has been notably illustrated by the use of a disulfide-containing ATRP inimer ${ }^{128}$ and by the combination of two monomers that polymerize by different chemistries (ROP and ATRP) and bear initiating centres for the opposite types of chemistry. This leads to a concurrent one-step polymerization process ${ }^{129}$. Degradability was also conferred to polymeric capsules formed by layer-by-layer assembly ${ }^{130}$ by means of degradable crosslinkers connecting together the different layers. This has been performed using a bisazide linker containing a disulfide bond, via copper(I)-catalysed alkyne-azide Huisgen cycloaddition, also termed 'click' chemistry ${ }^{131}$. This way, deconstruction of the capsules is expected to occur through exposure to a reducing environment, similar to that found in the intracellular cytosolic space. The concept has been further extended to the design of subcompartimentalized layer-by-layer capsules with selectively degradable carriers and subunits in response to multiple chemical stimuli. This was achieved simply by implementing degradable crosslinkers with different labile functionalities (for example, disulfide bond, vicinal diol group $)^{132}$.

\section{Cleavable bioconjugates}

Although on the fringe of degradable vinyl materials, polymer chain cleavage from organic substrates can also be seen as a form of degradability. This concept takes on its full meaning in the case of biological substrates, such as therapeutic proteins or peptides. Covalent attachment of PEG to proteins, termed PEGylation, confers indisputable pharmacological advantages to native proteins, such as improved solubility and stability, extended circulation time, and reduced proteolytic degradation ${ }^{133}$. However, their biological activity is often decreased because of stable polymer attachment. Therefore, it is desirable to efficiently release the protein therapeutics from the
PEG chains to enhance their biological performances. Also, if the molecular weight of the released polymer is below the renal filtration threshold, its complete removal from the body will likely occur, which will not be the case for a stable linkage.

The 'grafting to' technique. Several linear PEGs have been attached to proteins via degradable linkages ${ }^{134}$, allowing for the gradual release of the target protein and thereby overcoming the normal limitation of PEGylation. One can also take advantage of CRP techniques to design $\alpha$-functional vinyl polymers for reversible protein bioconjugation ${ }^{135}$. In this sense, disulfide linkages have been advantageously utilized in several proofs of concept. A common strategy is to prepare pyridyl disulfide (PDS) end-terminated polymers for subsequent coupling under mild conditions with free cysteine-containing biological macromolecules, such as proteins (Fig. 9) ${ }^{136,137}$ or siRNA ${ }^{138}$. Owing to the flexibility of CRP, the PDS moiety can also be positioned at the polymer mid-chain leading to branched bioconjugates with an expected improvement in masking the protein surface due to the so-called umbrella-like effect ${ }^{139}$. For targeting lysine residues, the disulfide functionality is incorporated in between a lysine-reactive moiety and the polymer chain ${ }^{140}$. This was illustrated by the design of thiazolidine-2-thione endterminated POEGMA via RAFT polymerization, further reacted with lysozyme, used as a model protein. After cleavage of the polymer chains from the conjugates in aqueous tris(2-carboxyethyl) phosphine hydrochloride solution, the released protein displayed a marked increase in bioactivity. Interestingly, when the polymer is obtained from the copolymerization of OEGMA and 5,6-benzo2-methylene-1,3-dioxepane by rROP, both reductive cleavage of the polymer chains from the protein and hydrolytic degradation of the polymer in the presence of base are observed ${ }^{141}$. This could be a valuable advantage compared with traditional PEGylation, as the polymer should be released from the protein and subsequently degraded, to allow for excretion of initially larger polymers. However, this promising combining strategy still requires validation in vivo.
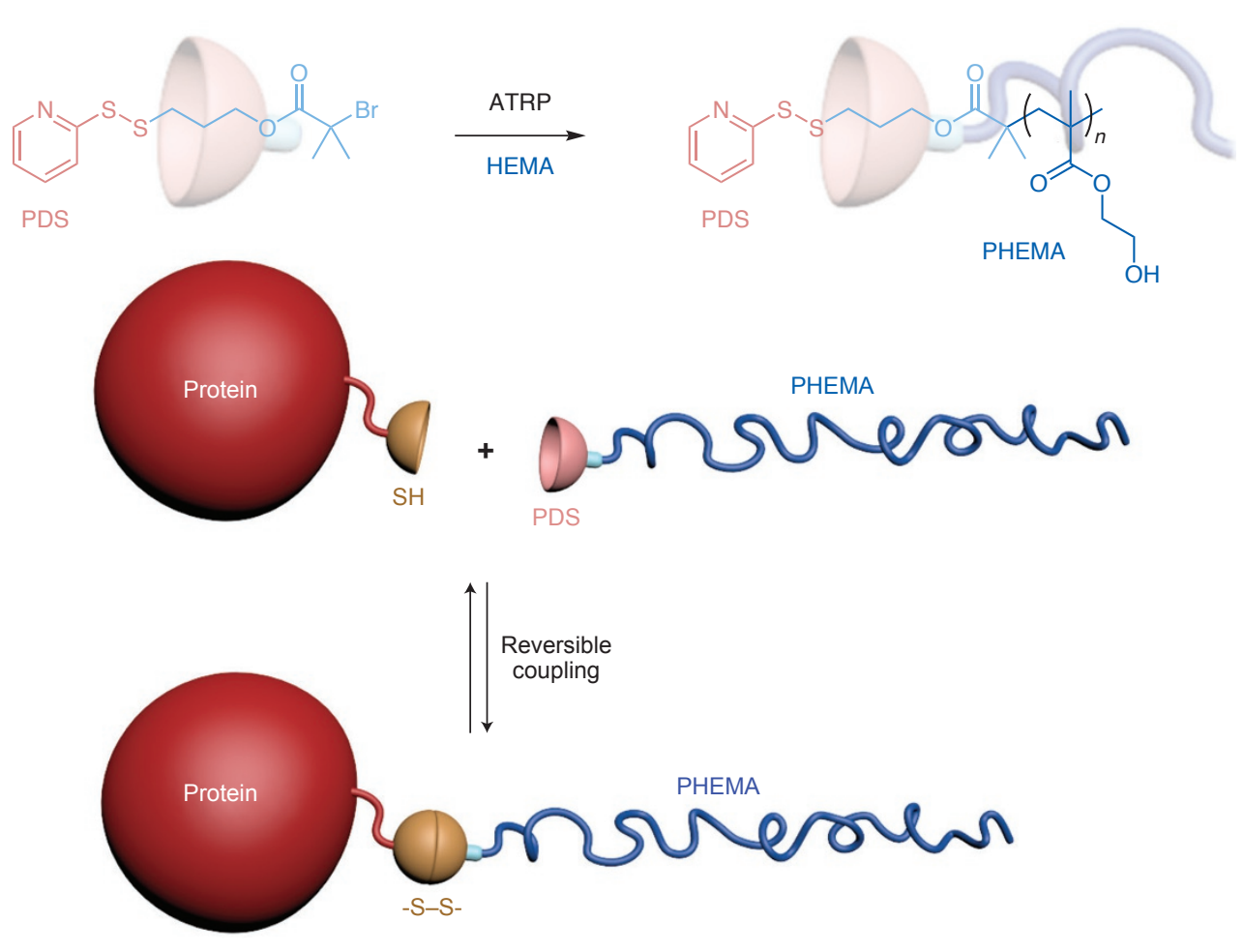

Figure 9 | Cleavable polymer-protein bioconjugates. Development of a bioreversible conjugation between cysteine-containing proteins and pyridyl disulfide (PDS) end-terminated PHEMA prepared by atom-transfer radical polymerization (ATRP) through the formation of a disulfide bond. 
Table 1 | Main features of the synthetic approaches to design degradable and biodegradable vinyl polymers.

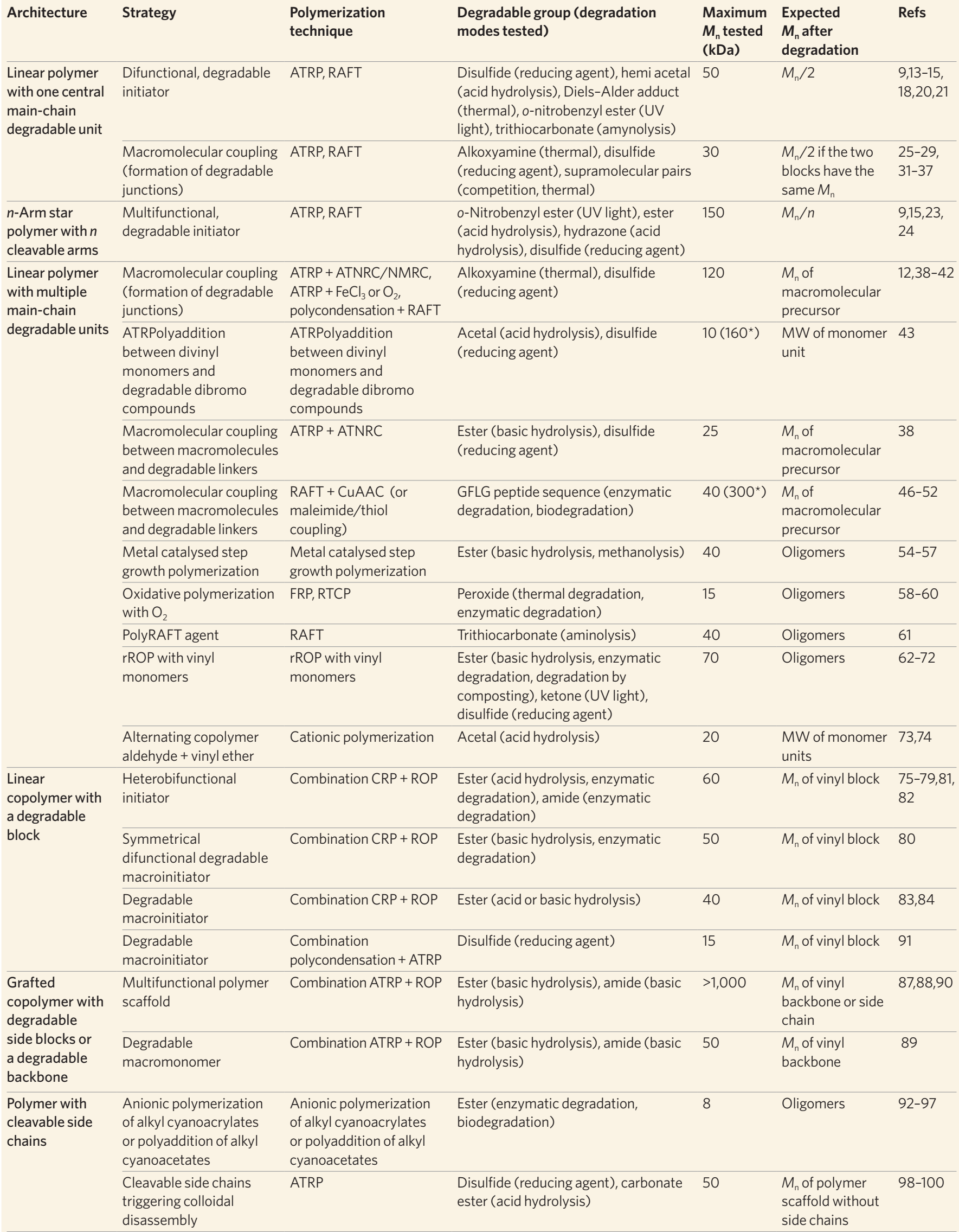




\begin{tabular}{|c|c|c|c|c|c|c|}
\hline Architecture & Strategy & $\begin{array}{l}\text { Polymerization } \\
\text { technique }\end{array}$ & $\begin{array}{l}\text { Degradable group (degradation } \\
\text { modes tested) }\end{array}$ & $\begin{array}{l}\text { Maximum } \\
M_{\mathrm{n}} \text { tested } \\
(\mathrm{kDa})\end{array}$ & $\begin{array}{l}\text { Expected } \\
M_{n} \text { after } \\
\text { degradation }\end{array}$ & Refs \\
\hline $\begin{array}{l}\text { Star polymer } \\
\text { with a } \\
\text { degradable } \\
\text { crosslinked core }\end{array}$ & $\begin{array}{l}\text { Degradable crosslinkers } \\
\text { in homogeneous media }\end{array}$ & ATRP + arm first method & Disulfide (reducing agent) & $>500$ & $M_{n}$ of arm & $\begin{array}{l}85,86 \\
101,102\end{array}$ \\
\hline \multirow{2}{*}{$\begin{array}{l}\text { Hyperbranched } \\
\text { polymer with } \\
\text { degradable } \\
\text { branching points }\end{array}$} & Degradable inimers & ATRP & Disulfide (reducing agent) & 5 & Oligomers & 128 \\
\hline & Degradable inimers & ROP + ATRP & Ester (acid hydrolysis) & 30 & Oligomers & 129 \\
\hline $\begin{array}{l}\text { Gel/hydrogel/ } \\
\text { microgel with } \\
\text { degradable } \\
\text { crosslinking } \\
\text { points }\end{array}$ & $\begin{array}{l}\text { Degradable crosslinkers } \\
\text { in homogeneous media }\end{array}$ & FRP, ATRP & $\begin{array}{l}\text { Ester (acid or basic hydrolysis), } \\
\text { disulfide (reducing agent), acetal (acid } \\
\text { hydrolysis), CYKC peptide sequence } \\
\text { (enzymatic degradation) }\end{array}$ & 120 & $\begin{array}{l}M_{n} \text { of primary } \\
\text { chain }\end{array}$ & $\begin{array}{l}80, \\
107-110, \\
112,114 \\
116\end{array}$ \\
\hline \multirow{2}{*}{$\begin{array}{l}\text { Crosslinked } \\
\text { particulate } \\
\text { system with } \\
\text { degradable } \\
\text { crosslinking } \\
\text { points }\end{array}$} & $\begin{array}{l}\text { Degradable crosslinkers } \\
\text { on preformed block } \\
\text { copolymer NPs }\end{array}$ & RAFT & Ketal (acid hydrolysis) & 15 & $\begin{array}{l}M_{n} \text { of block } \\
\text { copolymer }\end{array}$ & 127 \\
\hline & $\begin{array}{l}\text { Crosslinking polymer } \\
\text { layers }\end{array}$ & RAFT + LbL & $\begin{array}{l}\text { Disulfide (reducing agent), vicinal diols } \\
\text { (reducing agent, oxidative agent) }\end{array}$ & n.d. & $\begin{array}{l}M_{\mathrm{n}} \text { of polymer } \\
\text { chain }\end{array}$ & 131,132 \\
\hline
\end{tabular}

ATNRC: atom-transfer nitroxide radical coupling; ATRP: atom-transfer radical polymerization; CRP: controlled radical polymerization; CuAAC: Copper(I)-catalysed alkyne-azide Huisgen cycloaddition; FRP: freeradical polymerization; LbL: layer-by-layer; $M_{n}$ : number average molar mass; $M W$ : molecular weight; $n . d$. : not determined; NMP: nitroxide-mediated polymerization; NMRC: nitroxide-mediated radical coupling; NPs: nanoparticles; RAFT: reversible addition-fragmentation chain-transfer; ROP: ring-opening polymerization; rROP: radical ring-opening polymerization; RTCP: reversible chain-transfer catalysed polymerization. ${ }^{\star}$ After fractionation.

The 'grafting from' technique. The polymer chain also can be grown from the substrate by grafting a PDS-based ATRP initiator ${ }^{142}$ or a RAFT agent ${ }^{143}$ before the polymerization step. This 'grafting from' approach offers valuable advantages compared with the 'grafting to' method. For instance, the efficiency of the conjugation should be nearly quantitative due to negligible steric hindrance and purification of the conjugate might be easier since only the unreacted monomers have to be removed, as opposed to a preformed polymer. Importantly, the conjugates also retain their activities compared to the unmodified model proteins, meaning that the polymerization process is not detrimental to the biological substrates ${ }^{142}$. Additional studies performed in vivo to evaluate the value added by such degradable linkage would be strongly beneficial to this area.

\section{Summary and outlook}

This Review has summarized the recent progress in the development of groundbreaking strategies to confer degradability to vinyl polymers - a feature of paramount importance especially in several biomedical applications and for sustainable development. The vast majority of these achievements have been made possible by harnessing the robustness and versatility of modern polymerization methods and/or efficient ligation strategies to incorporate discrete or multiple cleavable functionalities. Beyond the degrability itself, which is crucial for toxicity concerns, intelligent placement of labile groups in a polymer structure can also trigger colloidal disassembly. This dual role is particularly relevant for drug delivery applications where the drug release from polymer nanocarriers should be governed by endogenous stimuli, such as $\mathrm{pH}$ or specific enzyme activity.

The main features of the synthetic approaches covered in this Review (except related to cleavable conjugates) are reported in Table 1. It includes the general strategies, the degradation modes tested, the highest molar masses investigated as well as the expected molar mass decrease after degradation, which gives an indication of the molar mass of the degradation products. Although the limits of these synthetic strategies have certainly not yet been reached, these data may be of great help for further synthetic developments to satisfy the requirements for other degradation modes, materials with higher molar mass or materials leading to lower molar mass degradation products. In general, the highest molar masses that have been tested ranged from several thousand to tens of thousands daltons, except for grafted and star polymers and macromolecular coupling approaches that enabled higher molar masses to be reached (typically above $100,000 \mathrm{Da}$ ). Combining radical polymerization and ROP from multifunctional polymer scaffolds enabled the preparation of higher molar mass materials (typically above 1 million $\mathrm{Da}$ ) whose degradability is, however, restricted to polyester chains and backbones. Another important benefit of using CRP techniques is the ability to fine-tune the molar mass of the vinyl polymer, leading to great flexibility with regards to the desired application.

Among the different options to introduce labile groups in vinyl polymers, rROP appears to be one of the most efficient methods, but for decades received only intermittent attention. In recent years, however, there has been a real resurgence of interest, which could be explained by the possibility to copolymerize traditional vinyl monomers with monomers that polymerize by rROP, either by conventional or controlled radical polymerization. The full potential of this technique has not yet been fully exploited. The diversity of suitable monomers is still rather poor and there is a crucial lack of hydrophilic and functionalized structures. In copolymerization, owing to unfavourable reactivity ratios, a large excess of these monomers is required to incorporate significant amounts in the resulting copolymer. This displays a lack of 'atom economy', which is becoming more and more important. Furthermore, their homopolymerization is not straightforward and often fails to be controlled. Thus, some work remains to be done to allow rROP to approach the versatility of ROP, which is 
still unparalleled in the biomedical context in terms of synthesis, properties, innocuousness and in vivo degradability.

It should be noted that the majority of vinyl polymers described in this Review (except PHPMA, PACA and rROP-derived polymers) have only been subjected to hydrolytic degradation (often accelerated in strongly acidic or basic environments), reductive degradation in vitro in the presence of reducing agents, or enzymatic degradation in vitro to prove their degradability. Yet, in vivo degradability or biodegradability is mandatory in the context of specific biomedical applications, such as drug delivery and tissue engineering. Although all these proofs of concept have already given solid indications of degradability, they must eventually be validated by undertaking appropriate in vivo degradability studies. This is crucial to discriminate between degradable materials that may find application in the biomedical field and those whose properties make them more appropriate for other types of applications (for example, nanolithography). Finally, future studies on newly developed degradable vinyl polymers should include in-depth investigations assessing their biocompatibility and the absence of toxicity, including those of their degradation products. These two major properties are often neglected, although they represent important steps to demonstrate the safety of these materials before any pharmaceutical or biomedical use.

Received 9 September 2014; accepted 4 August 2015; published online 22 September 2015

\section{References}

1. Plastics - the Facts 2014/2015 (PlasticsEurope, 2015); http://www.plasticseurope.org/Document/plastics-the-facts-20142015.aspx

2. Chiellini, E., Corti, A., D’Antone, S. \& Solaro, R. Biodegradation of poly(vinyl alcohol) based materials. Prog. Polym. Sci. 28, 963-1014 (2003).

3. Nicolas, J. et al. Nitroxide-mediated polymerization. Prog. Polym. Sci. 38, 63-235 (2013).

4. Matyjaszewski, K. \& Xia, J. Atom transfer radical polymerization. Chem. Rev. 101, 2921-2990 (2001).

5. Kamigaito, M., Ando, T. \& Sawamoto, M. Metal-catalyzed living radical polymerization. Chem. Rev. 101, 3689-3745 (2001).

6. Moad, G., Rizzardo, E. \& Thang, S. H. Living radical polymerization by the RAFT process - a second update. Aust. J. Chem. 62, 1402-1472 (2009).

7. Vert, M. et al. Terminology for biorelated polymers and applications (IUPAC recommendations 2012). Pure Appl. Chem. 84, 377-408 (2012).

8. Pasut, G. \& Veronese, F. M. Polymer-drug conjugation, recent achievements and general strategies. Prog. Polym. Sci. 32, 933-961 (2007).

9. Rikkou, M. D. \& Patrickios, C. S. Polymers prepared using cleavable initiators: synthesis, characterization and degradation. Prog. Polym. Sci. 36, 1079-1097 (2011)

10. Xu, H., Cao, W. \& Zhang, X. Selenium-containing polymers: promising biomaterials for controlled release and enzyme mimics. Acc. Chem. Res. 46, 1647-1658 (2013).

11. Phillips, D. J. \& Gibson, M. I. Redox-sensitive materials for drug delivery: targeting the correct intracellular environment, tuning release rates, and appropriate predictive systems. Antioxid. Redox Sign. 21, 786-803 (2013).

12. Tsarevsky, N. V. \& Matyjaszewski, K. Reversible redox cleavage/coupling of polystyrene with disulfide or thiol groups prepared by atom transfer radical polymerization. Macromolecules 35, 9009-9014 (2002).

13. Syrett, J. A., Mantovani, G., Barton, W. R. S., Price, D. \& Haddleton, D. M. Self-healing polymers prepared via living radical polymerisation. Polym. Chem. 1, 102-106 (2010).

14. Rikkou-Kalourkoti, M., Loizou, E., Porcar, L., Matyjaszewski, K. \& Patrickios, C. S. End-linked, amphiphilic, degradable polymer conetworks: synthesis by sequential atom transfer radical polymerization using a bifunctional, cleavable initiator. Polym. Chem. 3, 105-116 (2012).

15. Johnson, J. A., Finn, M. G., Koberstein, J. T. \& Turro, N. J. Synthesis of photocleavable linear macromonomers by ATRP and star macromonomers by a tandem ATRP-click reaction:precursors to photodegradable model networks. Macromolecules 40, 3589-3598 (2007)

16. Schumers, J-M., Gohy, J-F. \& Fustin, C-A. A versatile strategy for the synthesis of block copolymers bearing a photocleavable junction. Polym. Chem. 1, 161-163 (2010).

17. Cabane, E., Malinova, V., Menon, S., Palivan, C. G. \& Meier, W. Photoresponsive polymersomes as smart, triggerable nanocarriers. Soft Matter 7, 9167-9176 (2011).
18. Ge, Z. et al. Facile synthesis of dumbbell-shaped dendritic-linear-dendritic triblock copolymer via reversible addition-fragmentation chain transfer polymerization. J. Polym. Sci. A 45, 1432-1445 (2007).

19. Vogt, A. P. \& Sumerlin, B. S. Temperature and redox responsive hydrogels from ABA triblock copolymers prepared by RAFT polymerization. Soft Matter 5, 2347-2351 (2009).

20. Madsen, J. et al. Biocompatible wound dressings based on chemically degradable triblock copolymer hydrogels. Biomacromolecules 9, 2265-2275 (2008)

21. Li, C., Madsen, J., Armes, S. P. \& Lewis, A. L. A new class of biochemically degradable, stimulus-responsive triblock copolymer gelators. Angew. Chem. Int. Ed. 118, 3510-3513 (2006).

22. Jiang, X., Chen, Y. \& Xi, F. Stepwise cleavable star polymers and polymeric gels thereof. Macromolecules 43, 7056-7061 (2010).

23. Chen, B. et al. Synthesis and properties of star-comb polymers and their doxorubicin conjugates. Bioconjugate Chem. 22, 617-624 (2011).

24. Durmaz, Y. Y., Lin, Y-L. \& ElSayed, M. E. H. Development of degradable, $\mathrm{pH}$-sensitive star vectors for enhancing the cytoplasmic delivery of nucleic acids. Adv. Funct. Mater. 23, 3885-3895 (2013)

25. Wong, E. H. H., Junkers, T. \& Barner-Kowollik, C. Nitrones in synthetic polymer chemistry. Polym. Chem. 2, 1008-1017 (2011).

26. Lin, W., Fu, Q., Zhang, Y. \& Huang, J. One-pot aynthesis of ABC type triblock copolymers via a combination of click chemistry and atom transfer nitroxide radical coupling chemistry. Macromolecules 41, 4127-4135 (2008).

27. Kulis, J., Bell, C. A., Micallef, A. S., Jia, Z. \& Monteiro, M. J. Rapid, selective, and reversible nitroxide radical coupling (NRC) reactions at ambient temperature. Macromolecules 42, 8218-8227 (2009).

28. Klaikherd, A., Ghosh, S. \& Thayumanavan, S. A facile method for the synthesis of cleavable block copolymers from ATRP-based homopolymers. Macromolecules 40, 8518-8520 (2007).

29. Klaikherd, A., Nagamani, C. \& Thayumanavan, S. Multi-stimuli sensitive amphiphilic block copolymer assemblies. J. Am. Chem. Soc 131, 4830-4838 (2009).

30. Yang, S. K., Ambade, A. V. \& Weck, M. Main-chain supramolecular block copolymers. Chem. Soc. Rev. 40, 129-137 (2011).

31. Sambe, L. et al. Multi-stimuli responsive supramolecular diblock copolymers. Polym. Chem. 5, 1031-1036 (2014).

32. Loh, X. J. et al. Triply triggered doxorubicin release from supramolecular nanocontainers. Biomacromolecules 13, 84-91 (2011).

33. Guo, M. \& Jiang, M. Non-covalently connected micelles (NCCMs): the origins and development of a new concept. Soft Matter 5, 495-500 (2009).

34. Moughton, A. O. \& O’Reilly, R. K. Noncovalently connected micelles, nanoparticles, and metal-functionalized nanocages using supramolecular selfassembly. J. Am. Chem. Soc. 130, 8714-8725 (2008)

35. Yang, S. K., Ambade, A. V. \& Weck, M. Supramolecular ABC triblock copolymers via one-pot, orthogonal self-assembly. J. Am. Chem. Soc. 132, 1637-1645 (2010)

36. Mura, S., Nicolas, J. \& Couvreur, P. Stimuli-responsive nanocarriers for drug delivery systems. Nature Mater. 12, 991-1003 (2013).

37. Yan, Q. et al. Voltage-responsive vesicles based on orthogonal assembly of two homopolymers. J. Am. Chem. Soc. 132, 9268-9270 (2010).

38. Nicolaÿ, R., Marx, L., Hémery, P. \& Matyjaszewski, K. Synthesis of multisegmented degradable polymers by atom transfer radical cross-coupling. Macromolecules 40, 9217-9223 (2007).

39. Wong, E. H. H., Stenzel, M. H., Junkers, T. \& Barner-Kowollik, C. Embedding multiple site-specific functionalities into polymer chains via nitrone-mediated radical coupling reactions. J. Polym. Sci. A 49, 2118-2126 (2011).

40. You, Y-Z., Manickam, D. S., Zhou, Q-H. \& Oupický, D. A Versatile approach to reducible vinyl polymers via oxidation of telechelic polymers prepared by reversible addition fragmentation chain transfer polymerization. Biomacromolecules 8, 2038-2044 (2007).

41. You, Y-Z. et al. Dually responsive multiblock copolymers via reversible addition-fragmentation chain transfer polymerization: synthesis of temperature- and redox-responsive copolymers of poly( $N$-isopropylacrylamide) and poly(2-(dimethylamino)ethyl methacrylate). Macromolecules 40, 8617-8624 (2007).

42. Phillips, D. J. \& Gibson, M. I. Biodegradable poly(disulfide)s derived from raft polymerization: monomer scope, glutathione degradation, and tunable thermal responses. Biomacromolecules 13, 3200-3208 (2012).

43. Zhang, L-J., Dong, B-T., Du, F-S. \& Li, Z-C. Degradable thermoresponsive polyesters by atom transfer radical polyaddition and click chemistry. Macromolecules 45, 8580-8587 (2012).

44. Kopeček, J. \& Kopečková, P. HPMA copolymers: origins, early developments, present, and future. Adv. Drug Deliver. Rev. 62, 122-149 (2010).

45. Doxorubicin in treating women with advanced breast cancer. ClinicalTrials.gov (1999); https://clinicaltrials.gov/ct2/show/NCT00003165 
46. Luo, K., Yang, J., Kopečková, P. \& Kopeček, J. Biodegradable multiblock poly[ $N$-(2-hydroxypropyl)methacrylamide] via reversible addition-fragmentation chain transfer polymerization and click chemistry. Macromolecules 44, 2481-2488 (2011).

47. Pan, H. et al. Efficiency of high molecular weight backbone degradable HPMA copolymer-Prostaglandin E1 conjugate in promotion of bone formation in ovariectomized rats. Biomaterials 34, 6528-6538 (2013).

48. Zhang, R. et al. Synthesis and evaluation of a backbone biodegradable multiblock HPMA copolymer nanocarrier for the systemic delivery of paclitaxel. J. Control. Rel. 166, 66-74 (2013).

49. Pan, H., Yang, J., Kopečková, P. \& Kopeček, J. Backbone degradable multiblock $\mathrm{N}$-(2-hydroxypropyl)methacrylamide copolymer conjugates via reversible addition-fragmentation chain transfer polymerization and thiol-ene coupling reaction. Biomacromolecules 12, 247-252 (2010).

50. Duangjai, A., Luo, K., Zhou, Y., Yang, J. \& Kopeček, J. Combination cytotoxicity of backbone degradable HPMA copolymer gemcitabine and platinum conjugates toward human ovarian carcinoma cells. Eur. J. Pharm. Biopharm. 87, 187-196 (2014).

51. Larson, N. et al. Biodegradable multiblock poly(N-2-hydroxypropyl) methacrylamide gemcitabine and paclitaxel conjugates for ovarian cancer cell combination treatment. Int. J. Pharm. 454, 435-443 (2013).

52. Pan, H., Sima, M., Yang, J. \& Kopeček, J. Synthesis of long-circulating, backbone degradable HPMA copolymer-doxorubicin conjugates and evaluation of molecular-weight-dependent antitumor efficacy. Macromol. Biosci. 13, 155-160 (2013).

53. Etrych, T. et al. Biodegradable star HPMA polymer-drug conjugates: biodegradability, distribution and anti-tumor efficacy. J. Control. Rel. 154, 241-248 (2011).

54. Mizutani, M., Satoh, K. \& Kamigaito, M. Metal-catalyzed simultaneous chain- and step-growth radical polymerization: marriage of vinyl polymers and polyesters. J. Am. Chem. Soc. 132, 7498-7507 (2010)

55. Mizutani, M., Satoh, K. \& Kamigaito, M. Metal-catalyzed radical polyaddition for aliphatic polyesters via evolution of atom transfer radical addition into step-growth polymerization. Macromolecules 42, 472-480 (2008).

56. Satoh, K., Ozawa, S., Mizutani, M., Nagai, K. \& Kamigaito, M. Sequenceregulated vinyl copolymers by metal-catalysed step-growth radical polymerization. Nature Commun. 1, 6 (2010).

57. Mizutani, M. et al. Design and synthesis of self-degradable antibacterial polymers by simultaneous chain- and step-growth radical copolymerization. Biomacromolecules 13, 1554-1563 (2012).

58. Matsumoto, A. \& Higashi, H. convenient synthesis of polymers containing labile bonds in the main chain by radical alternating copolymerization of alkyl sorbates with oxygen. Macromolecules 33, 1651-1655 (2000).

59. Sato, E., Hagihara, T. \& Matsumoto, A. Facile synthesis of main-chain degradable block copolymers for performance enhanced dismantlable adhesion. ACS Appl. Mater. Interfaces 4, 2057-2064 (2012).

60. Pal, S., Das, A., Maiti, S. \& De, P. Synthesis and characterization of a biodegradable polymer prepared viaradical copolymerization of 2-(acetoacetoxy)ethyl methacrylate and molecular oxygen. Polym. Chem. 3, 182-189 (2012).

61. You, Y-Z., Hong, C-Y. \& Pan, C-Y. A novel strategy for synthesis of multiblock copolymers. Chem. Commun. 2800-2801 (2002).

62. Bailey, W. J., Ni, Z. \& Wu, S. R. Free radical ring-opening polymerization of 4,7-dimethyl-2-methylene-1,3-dioxepane and 5,6-benzo-2-methylene-1,3dioxepane. Macromolecules 15, 711-714 (1982).

63. Agarwal, S. Chemistry, chances and limitations of the radical ring-opening polymerization of cyclic ketene acetals for the synthesis of degradable polyesters. Polym. Chem. 1, 953-964 (2010).

64. Lutz, J-F., Andrieu, J., Üzgün, S., Rudolph, C. \& Agarwal, S. Biocompatible, thermoresponsive, and biodegradable: a simple preparation of all-in-one biorelevant polymers. Macromolecules 40, 8540-8543 (2007).

65. Delplace, V. et al. Degradable and comblike PEG-based copolymers by nitroxide-mediated radical ring-opening polymerization. Biomacromolecules 14, 2837-2847 (2013)

66. Siegwart, D. J., Bencherif, S. A., Srinivasan, A., Hollinger, J. O. \& Matyjaszewski, K. Synthesis, characterization, and in vitro cell culture viability of degradable poly $(N$-isopropylacrylamide-co-5,6-benzo2-methylene-1,3-dioxepane)-based polymers and crosslinked gels. J. Biomed. Mater. Res. A 87, 345-358 (2008).

67. Agarwal, S. \& Ren, L. Polycaprolactone-based novel degradable ionomers by radical ring-opening polymerization of 2-methylene-1,3-dioxepane. Macromolecules 42, 1574-1579 (2009).

68. Riachi, C., Schüwer, N. \& Klok, H-A. Degradable polymer brushes prepared via surface-initiated controlled radical polymerization. Macromolecules 42, 8076-8081 (2009)

69. Undin, J., Finne-Wistrand, A. \& Albertsson, A-C. Copolymerization of 2-methylene-1,3-dioxepane and glycidyl methacrylate, a well-defined and efficient process for achieving functionalized polyesters for covalent binding of bioactive molecules. Biomacromolecules 14, 2095-2102 (2013).

70. Ishikawa, T., Morino, K., Sudo, A. \& Endo, T. Synthesis of photo-scissible poly( $p$-hydroxystyrene) derivatives by radical copolymerization of p-hydroxystyrene derivatives and methyl vinyl ketone. J. Polym. Sci. A 49, 4714-4720 (2011)

71. Evans, R. A. \& Rizzardo, E. Free-radical ring-opening polymerization of cyclic allylic sulfides. Macromolecules 29, 6983-6989 (1996).

72. Paulusse, J. M. J., Amir, R. J., Evans, R. A. \& Hawker, C. J. Free radical polymers with tunable and selective bio- and chemical degradability. J. Am. Chem. Soc. 131, 9805-9812 (2009).

73. Ishido, Y., Kanazawa, A., Kanaoka, S. \& Aoshima, S. New degradable alternating copolymers from naturally occurring aldehydes: well-controlled cationic copolymerization and complete degradation. Macromolecules 45, 4060-4068 (2012)

74. Aoshima, S. et al. Efficient design for stimuli-responsive polymers with quantitative acid-degradability: specifically designed alternating controlled cationic copolymerization and facile complete degradation. ACS Macro Lett. 3, 80-85 (2013)

75. Habraken, G. J. M., Peeters, M., Thornton, P. D., Koning, C. E. \& Heise, A. Selective enzymatic degradation of self-assembled particles from amphiphilic block copolymers obtained by the combination of $\mathrm{N}$-carboxyanhydride and nitroxide-mediated polymerization. Biomacromolecules 12, 3761-3769 (2011)

76. Ko, N. R., Yao, K., Tang, C. \& Oh, J. K. Synthesis and thiol-responsive degradation of polylactide-based block copolymers having disulfide junctions using ATRP and ROP. J. Polym. Sci. A 51, 3071-3080 (2013).

77. Li, J. et al. Stimuli-responsive zwitterionic block copolypeptides: poly( $\mathrm{N}$-isopropylacrylamide)-block-poly(lysine-co-glutamic acid). Biomacromolecules 9, 2670-2676 (2008).

78. Jacobs, J., Gathergood, N. \& Heise, A. Synthesis of polypeptide block copolymer hybrids by the combination of $\mathrm{N}$-carboxyanhydride polymerization and RAFT. Macromol. Rapid Commun. 34, 1325-1329 (2013).

79. Mecerreyes, D. et al. Simultaneous dual living polymerizations: a novel one-step approach to block and graft copolymers. Angew. Chem. Int. Ed. 37, 1274-1276 (1998)

80. Atzet, S., Curtin, S., Trinh, P., Bryant, S. \& Ratner, B. Degradable poly(2-hydroxyethyl methacrylate)-co-polycaprolactone hydrogels for tissue engineering scaffolds. Biomacromolecules 9, 3370-3377 (2008).

81. Xu, F. J. et al. Thermo-responsive porous membranes of controllable porous morphology from triblock copolymers of polycaprolactone and $\operatorname{poly}(\mathrm{N}$-isopropylacrylamide) prepared by atom transfer radical polymerization. Biomacromolecules 9, 331-339 (2007).

82. Jubeli, E., Moine, L. \& Barratt, G. Synthesis, characterization, and molecular recognition of sugar-functionalized nanoparticles prepared by a combination of ROP, ATRP, and click chemistry. J. Polym. Sci. A 48, 3178-3187 (2010).

83. Barz, M. et al. P(HPMA)-block-P(LA) copolymers in paclitaxel formulations: polylactide stereochemistry controls micellization, cellular uptake kinetics, intracellular localization and drug efficiency. J. Control. Rel. 163, 63-74 (2012).

84. Zhang, Q., Remsen, E. E. \& Wooley, K. L. Shell cross-linked nanoparticles containing hydrolytically degradable, crystalline core domains. J. Am. Chem. Soc. 122, 3642-3651 (2000).

85. Wiltshire, J. T. \& Qiao, G. G. Selectively degradable core cross-linked star polymers. Macromolecules 39, 9018-9027 (2006).

86. Wiltshire, J. T. \& Qiao, G. G. Synthesis of core cross-linked star polymers with adjustable coronal properties. Macromolecules 41, 623-631 (2007)

87. Liu, Y., Chen, P. \& Li, Z. Molecular bottlebrushes with polypeptide backbone prepared via ring-opening polymerization of NCA and ATRP. Macromol. Rapid Commun. 33, 287-295 (2012).

88. Lee, H-i., Matyjaszewski, K., Yu-Su, S. \& Sheiko, S. S. Hetero-grafted block brushes with PCL and PBA side chains. Macromolecules 41, 6073-6080 (2008).

89. Hawker, C. J. et al. Living free radical polymerization of macromonomers: preparation of well defined graft copolymers. Macromol. Chem. Phys. 198, 155-166 (1997)

90. Lee, H-i., Jakubowski, W., Matyjaszewski, K., Yu, S. \& Sheiko, S. S. Cylindrical core-shell brushes prepared by a combination of ROP and ATRP. Macromolecules 39, 4983-4989 (2006)

91. Aleksanian, S., Khorsand, B., Schmidt, R. \& Oh, J. K. Rapidly thiol-responsive degradable block copolymer nanocarriers with facile bioconjugation. Polym. Chem. 3, 2138-2147 (2012)

92. Nicolas, J. \& Couvreur, P. Synthesis of poly(alkyl cyanoacrylate)-based colloidal nanomedicines. Wiley Interdiscip. Rev. Nanomed. Nanobiotechnol. 1, 111-127 (2009).

93. Lenaerts, V. et al. Degradation of poly(isobutyl cyanoacrylate) nanoparticles. Biomaterials 5, 65-68 (1984). 
94. Efficacy and safety doxorubicin transdrug study in patients suffering from advanced hepatocellular carcinoma (ReLive) ClinicalTrials.gov (2012); https://clinicaltrials.gov/ct2/show/NCT01655693

95. Brambilla, D. et al. Design of fluorescently tagged poly(alkyl cyanoacrylate) nanoparticles for human brain endothelial cell imaging. Chem. Commun. 46, 2602-2604 (2010).

96. Brambilla, D. et al. PEGylated nanoparticles bind to and alter amyloid-beta peptide conformation: toward engineering of functional nanomedicines for Alzheimer's disease. ACS Nano 6, 5897-5908 (2012).

97. Le Droumaguet, B. et al. Versatile and efficient targeting from a single nanoparticulate platform: application to cancer and Alzheimer's disease. ACS Nano 6, 5866-5879 (2012).

98. Khorsand, B., Lapointe, G., Brett, C. \& Oh, J. K. Intracellular drug delivery nanocarriers of glutathione-responsive degradable block copolymers having pendant disulfide linkages. Biomacromolecules 14, 2103-2111 (2013).

99. Jiang, X., Lok, M. C. \& Hennink, W. E. Degradable-brushed pHEMA-pDMAEMA synthesized via ATRP and click chemistry for gene delivery. Bioconjugate Chem. 18, 2077-2084 (2007)

100. Liu, J. et al. Reduction biodegradable brushed PDMAEMA derivatives synthesized by atom transfer radical polymerization and click chemistry for gene delivery. Acta Biomater. 9, 7758-7766 (2013).

101.Gao, H., Tsarevsky, N. V. \& Matyjaszewski, K. Synthesis of degradable miktoarm star copolymers via atom transfer radical polymerization. Macromolecules 38, 5995-6004 (2005).

102. Cho, H. Y. et al. Synthesis of biocompatible PEG-based star polymers with cationic and degradable core for siRNA delivery. Biomacromolecules 12, 3478-3486 (2011).

103. Li, Y. \& Armes, S. P. Synthesis and chemical degradation of branched vinyl polymers prepared via ATRP: use of a cleavable disulfide-based branching agent. Macromolecules 38, 8155-8162 (2005)

104. Rosselgong, J., Armes, S. P., Barton, W. \& Price, D. Synthesis of highly branched methacrylic copolymers: observation of near-ideal behavior using RAFT polymerization. Macromolecules 42, 5919-5924 (2009).

105. Wang, L., Li, C., Ryan, A. J. \& Armes, S. P. Synthesis and peptideinduced degradation of biocompatible fibers based on highly branched poly(2-hydroxyethyl methacrylate). Adv. Mater. 18, 1566-1570 (2006).

106. Mabilleau, G., Moreau, M. F., Filmon, R., Baslé, M. F. \& Chappard, D. Biodegradability of poly(2-hydroxyethyl methacrylate) in the presence of the J774.2 macrophage cell line. Biomaterials 25, 5155-5162 (2004)

107. Mespouille, L. et al. Novel biodegradable adaptive hydrogels: controlled synthesis and full characterization of the amphiphilic co-networks. Chem. Eur. J. 14, 6369-6378 (2008).

108. Ejaz, M. et al. Evaluation of redox-responsive disulfide cross-linked poly(hydroxyethyl methacrylate) hydrogels. Polymer 52, 5262-5270 (2011)

109. Murthy, N., Thng, Y. X., Schuck, S., Xu, M. C. \& Fréchet, J. M. J. A novel strategy for encapsulation and release of proteins: hydrogels and microgels with acid-labile acetal cross-linkers. J. Am. Chem. Soc. 124, 12398-12399 (2002).

110. Tsarevsky, N. V. \& Matyjaszewski, K. Combining atom transfer radical polymerization and disulfide/thiol redox chemistry: a route to well-defined (bio)degradable polymeric materials. Macromolecules 38, 3087-3092 (2005).

111. Ercole, F., Thissen, H., Tsang, K., Evans, R. A. \& Forsythe, J. S. Photodegradable hydrogels made via RAFT. Macromolecules 45, 8387-8400 (2012).

112. Galperin, A., Long, T. J., Garty, S. \& Ratner, B. D. Synthesis and fabrication of a degradable poly( $N$-isopropyl acrylamide) scaffold for tissue engineering applications. J. Biomed. Mater. Res. A 101, 775-786 (2013).

113. Bencherif, S. A. et al. Nanostructured hybrid hydrogels prepared by a combination of atom transfer radical polymerization and free radical polymerization. Biomaterials 30, 5270-5278 (2009).

114. Plunkett, K. N., Berkowski, K. L. \& Moore, J. S. Chymotrypsin responsive hydrogel: application of a disulfide exchange protocol for the preparation of methacrylamide containing peptides. Biomacromolecules 6, 632-637 (2005).

115. Martens, P. J., Bryant, S. J. \& Anseth, K. S. Tailoring the degradation of hydrogels formed from multivinyl poly(ethylene glycol) and poly(vinyl alcohol) macromers for cartilage tissue engineering. Biomacromolecules 4, 283-292 (2003)

116. Browning, M. B. \& Cosgriff-Hernandez, E. Development of a biostable replacement for PEGDA hydrogels. Biomacromolecules 13, 779-786 (2012).

117. Murthy, N. et al. A macromolecular delivery vehicle for protein-based vaccines: acid-degradable protein-loaded microgels. Proc. Natl Acad. Sci. USA 100, 4995-5000 (2003).

118. Standley, S. M. et al. Acid-degradable particles for protein-based vaccines: enhanced survival rate for tumor-challenged mice using ovalbumin model. Bioconjugate Chem. 15, 1281-1288 (2004).

119. Kwon, Y. J., Standley, S. M., Goodwin, A. P., Gillies, E. R. \& Fréchet, J. M. J. Directed antigen presentation using polymeric microparticulate carriers degradable at lysosomal $\mathrm{pH}$ for controlled immune responses. Mol. Pharm. 2, 83-91 (2005).
120. Goh, S. L., Murthy, N., Xu, M. \& Fréchet, J. M. J. Cross-linked microparticles as carriers for the delivery of plasmid DNA for vaccine development. Bioconjugate Chem. 15, 467-474 (2004).

121. Standley, S. M. et al. Incorporation of CpG oligonucleotide ligand into protein-loaded particle vaccines promotes antigen-specific CD8 T-cell immunity. Bioconjugate Chem. 18, 77-83 (2006).

122. Beaudette, T. T. et al. In vivo studies on the effect of co-encapsulation of CpG DNA and antigen in acid-degradable microparticle vaccines. Mol. Pharm. 6, 1160-1169 (2009)

123. Cohen, J. L. et al. Enhanced cell penetration of acid-degradable particles functionalized with cell-penetrating peptides. Bioconjugate Chem. 19, 876-881 (2008).

124. Oh, J. K., Tang, C., Gao, H., Tsarevsky, N. V. \& Matyjaszewski, K. Inverse miniemulsion ATRP: a new method for synthesis and functionalization of well-defined water-soluble/cross-linked polymeric particles. J. Am. Chem. Soc 128, 5578-5584 (2006).

125. Oh, J. K., Siegwart, D. J. \& Matyjaszewski, K. Synthesis and biodegradation of nanogels as delivery carriers for carbohydrate drugs. Biomacromolecules 8, 3326-3331 (2007).

126. Oh, J. K. et al. Biodegradable nanogels prepared by atom transfer radical polymerization as potential drug delivery carriers: synthesis, biodegradation, in vitro release, and bioconjugation. J. Am. Chem. Soc. 129, 5939-5945 (2007).

127. Duong, H. T. T., Marquis, C. P., Whittaker, M., Davis, T. P. \& Boyer, C. Acid degradable and biocompatible polymeric nanoparticles for the potential codelivery of therapeutic agents. Macromolecules 44, 8008-8019 (2011).

128. Tsarevsky, N. V., Huang, J. \& Matyjaszewski, K. Synthesis of hyperbranched degradable polymers by atom transfer radical (co)polymerization of inimers with ester or disulfide groups. J. Polym. Sci. A 47, 6839-6851 (2009).

129. Mecerreyes, D., Trollsås, M. \& Hedrick, J. L. ABC BCD polymerization: a selfcondensing vinyl and cyclic ester polymerization by combination free-radical and ring-opening techniques. Macromolecules 32, 8753-8759 (1999).

130. Becker, A. L., Johnston, A. P. R. \& Caruso, F. Layer-by-layer-assembled capsules and films for therapeutic delivery. Small 6, 1836-1852 (2010)

131. Kinnane, C. R. et al. Low-fouling poly( $N$-vinyl pyrrolidone) capsules with engineered degradable properties. Biomacromolecules 10, 2839-2846 (2009).

132. Kulygin, O. et al. Subcompartmentalized polymer hydrogel capsules with selectively degradable carriers and subunits. Small 6, 1558-1564 (2010).

133. Duncan, R. The dawning era of polymer therapeutics. Nature Rev. Drug Discov. 2, 347-360 (2003).

134. Filpula, D. \& Zhao, H. Releasable PEGylation of proteins with customized linkers. Adv. Drug Deliver. Rev. 60, 29-49 (2008).

135. Le Droumaguet, B. \& Nicolas, J. Recent advances in the design of bioconjugates from controlled/living radical polymerization. Polym. Chem. 1, 563-598 (2010).

136. Boyer, C. et al. Direct synthesis of well-defined heterotelechelic polymers for bioconjugations. Macromolecules 41, 5641-5650 (2008)

137. Bontempo, D., Heredia, K. L., Fish, B. A. \& Maynard, H. D. Cysteine-reactive polymers synthesized by atom transfer radical polymerization for conjugation to proteins. J. Am. Chem. Soc. 126, 15372-15373 (2004)

138. Heredia, K. L. et al. Reversible siRNA-polymer conjugates by RAFT polymerization. Chem. Commun. 3245-3247 (2008).

139. Tao, L., Liu, J. \& Davis, T. P. Branched polymer-protein conjugates made from mid-chain-functional P(HPMA). Biomacromolecules 10, 2847-2851 (2009).

140. Tao, L., Liu, J., Hu, J. \& Davis, T. P. Bio-reversible polyPEGylation. Chem. Commun. 6560-6562 (2009).

141. Decker, C. G. \& Maynard, H. D. Degradable PEGylated protein conjugates utilizing RAFT polymerization. Eur. Polym. J. 65, 305-312 (2015).

142. Heredia, K. L. et al. In situ preparation of protein-"smart" polymer conjugates with retention of bioactivity. J. Am. Chem. Soc. 127, 16955-16960 (2005).

143. Liu, J. et al. In situ formation of protein-polymer conjugates through reversible addition fragmentation chain transfer polymerization. Angew. Chem. Int. Ed. 46, 3099-3103 (2007).

\section{Acknowledgements}

Some results described in this Review were obtained thanks to the French National Research Agency (ANR-11-JS08-0005), which founded the PhD of V.D. and from the European Community's Seventh Framework Programme (FP7/2007-2013) under agreement no. 212043. CNRS and Université Paris-Sud are also acknowledged for financial support.

\section{Additional information}

Reprints and permissions information is available online at www.nature.com/reprints. Correspondence and requests for materials should be addressed to J.N

\section{Competing financial interests}

The authors declare no competing financial interests. 